To appear in the Astrophysical Journal

\title{
Magnetic Field Structure of the HH 1-2 Region: Near-Infrared Polarimetry of Point-Like Sources
}

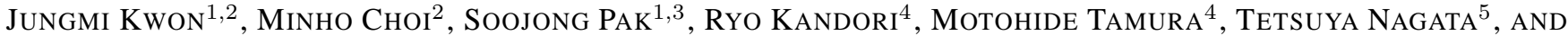 \\ SHUJI SATO 6 \\ ${ }^{1}$ Department of Astronomy and Space Science, Kyung Hee University, Yongin-si, Gyeonggi-do 446-701, South Korea \\ ${ }^{2}$ International Center for Astrophysics, Korea Astronomy and Space Science Institute, Daedukdaero 838, Yuseong, Daejeon 305-348, South Korea \\ 3 soojong@khu.ac.kr \\ ${ }^{4}$ National Astronomical Observatory of Japan, Mitaka, Tokyo 181-8588, Japan \\ ${ }^{5}$ Department of Astronomy, Kyoto University, Kyoto 606-8502, Japan \\ ${ }^{6}$ Department of Astrophysics, Nagoya University, Nagoya 464-8602, Japan
}

\begin{abstract}
The HH 1-2 region in the L1641 molecular cloud was observed in the near-IR $J, H$, and $K_{s}$ bands, and imaging polarimetry was performed. Seventy six point-like sources were detected in all three bands. The near-IR polarizations of these sources seem to be caused mostly by the dichroic extinction. Using a color-color diagram, reddened sources with little infrared excess were selected to trace the magnetic field structure of the molecular cloud. The mean polarization position angle of these sources is about $111^{\circ}$, which is interpreted as the projected direction of the magnetic field in the observed region of the cloud. The distribution of the polarization angle has a dispersion of about $11^{\circ}$, which is smaller than what was measured in previous studies. This small dispersion gives a rough estimate of the strength of the magnetic field to be about $130 \mu \mathrm{G}$ and suggests that the global magnetic field in this region is quite regular and straight. In contrast, the outflows driven by young stellar objects in this region seem to have no preferred orientation. This discrepancy suggests that the magnetic field in the L1641 molecular cloud does not dictate the orientation of the protostars forming inside.
\end{abstract}

Subject headings: infrared: stars — ISM: individual (HH 1-2) — ISM: structure — polarization — stars: formation

\section{INTRODUCTION}

Magnetic fields play a crucial role in various astrophysical processes, including the evolution of interstellar molecular clouds and star formation (Shu et al. 1987; Bergin \& Tafalla 2007; McKee \& Ostriker 2007). One of the problems related to star formation concerns the competition between magnetic and turbulent forces (Mac Low \& Klessen 2004). The magnetic field direction can be measured by observing the dichroic polarization of background stars in the optical and near-IR bands and/or the linearly polarized emission from the dust grains in the mid-IR and far-IR bands (Davis \& Greenstein 1951; Matthews \& Wilson 2000). The large-scale alignment of dust grains with the magnetic field is known to be the cause of the dichroic extinction and the interstellar polarization seen in the direction of background sources. Because of the low extinction, near-IR imaging polarimetry is particularly useful in tracing the dichroic polarization of background stars and embedded sources seen through dense clouds (Vrba et al. 1976; Wilking et al. 1979; Tamura et al. 1987; Kandori et al. 2007). Since both dichroic extinction and scattering processes can contribute to the polarization of embedded sources, multiwavelength polarimetry can be useful in discriminating between the two mechanisms (Casali 1995).

The L1641 cloud is one of the nearest giant molecular clouds and is a site of active star formation (Kutner et al. 1977; Maddalena et al. 1986; Strom et al. 1989; Morgan \& Bally 1991; Sakamoto et al. 1997; Zavagno et al. 1997). The role of magnetic field in the star formation activity of L1641 is complicated. With visual polarimetry of background stars, Vrba et al. (1988) found that the dispersion in position angles is large $\left(33^{\circ}\right)$ and suggested that the role of magnetic field in the global scale is only incidental. However, they also found that the outflows in L1641 tend to be parallel to the field direction and suggested that the role of magnetic field is important in the local scale. In contrast, Casali (1995) performed near-IR polarimetry of young stellar objects (YSOs) in L1641 and found that the alignment of polarization vectors is poor, which suggests that the magnetic field was not dominant in the collapse dynamics. To understand the situation better, it was suggested that more extensive polarimetry in the vicinity of each outflow and YSO is necessary (Vrba et al. 1988).

One of the well studied parts of the L1641 cloud is the region around the reflection nebula NGC 1999 and the HerbigHaro objects HH 1-2. This region contains several YSOs and outflows (Herbig 1951; Haro 1952; Warren-Smith et al. 1980; Strom et al. 1989; Corcoran \& Ray 1995; Choi \& Zhou 1997; Rodríguez et al. 2000). The magnetic field structure in the HH 1-2 region has been studied based on optical polarizations of point sources (Strom et al. 1985; Warren-Smith \& Scarrott 1999 , hereafter WS). They found that the local magnetic field is directed roughly along the axis of the HH 1-2 outflow, but the number of detectable stars was too small because of the large obscuration in this region.

In this paper, we present a wide-field near-IR polarimetry of the HH 1-2 region. In Section 2 we describe the observations and data reduction. In Section 3 we present the results of the polarimetry of point-like sources. In Section 4 we discuss the magnetic field structure and the star-forming activity in the $\mathrm{HH}$ 1-2 region. A summary is given in Section 5.

\section{OBSERVATIONS}

The observations toward the HH 1-2 region were carried out using the SIRPOL imaging polarimeter on the Infrared Survey Facility (IRSF) $1.4 \mathrm{~m}$ telescope at the South African Astronomical Observatory. SIRPOL consists of a single-beam polarimeter (an achromatic half-wave plate rotator unit and a polarizer) and an imaging camera (Nagayama et al. 2003). The camera, SIRIUS, has three $1024 \times 1024 \mathrm{HgCdTe}$ infrared detectors. IRSF/SIRPOL enables deep and wide-field $(7 ! 7 \times 7$ ! 7 with a scale of 0.45 pixel $^{-1}$ ) imaging polarimetry at the $J, H$, and $K_{s}$ 


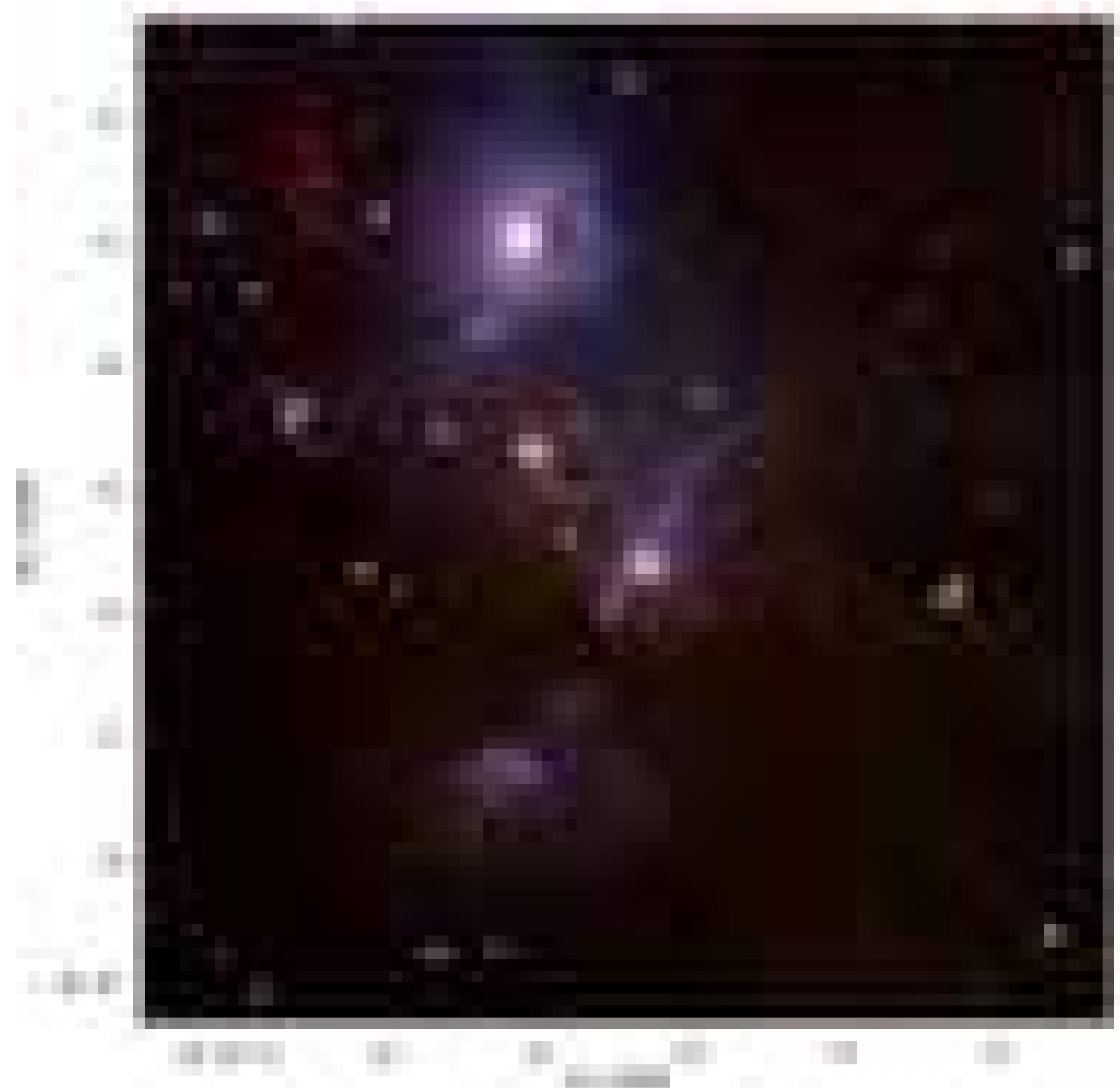

[See http://minho.kasi.re.kr/Publications.html for the original high-quality figure.]

Fig. 1.- Color composite Stokes $I$ image of the HH 1-2 region in the $J($ blue $), H($ green $)$, and $K_{s}($ red $)$ bands from the IRSF/SIRPOL observations.

bands simultaneously (Kandori et al. 2006).

The observations were made on the night of 2008 January 9. We performed $20 \mathrm{~s}$ exposures at 4 wave-plate angles (in the sequence of $0^{\circ}, 45^{\circ}, 22^{\circ} .5$, and 67.5 ) at 10 dithered positions for each set. The same observation sets were repeated 10 times toward the target object and the sky backgrounds for a better signal-to-noise ratio. The total integration time was $2000 \mathrm{~s}$ per wave plate angle. The typical seeing size during the observations was $\sim 1^{\prime \prime} 3$ in the $J$ band. The polarization efficiencies of SIRPOL are stable over several years, and the instrumental polarization is negligible (Kandori et al. 2006). The efficiencies were measured in 2007 December during a maintenance period, just a few days before our observing run, and were the same as the values reported by Kandori et al. (2006).

The data were processed using IRAF in the same manner as described by Kandori et al. (2006), which included darkfield subtraction, flat-field correction, median sky subtraction, and frame registration. Figure 1 shows the $J-H-K_{s}$ color composite intensity image of the $8^{\prime} \times 8^{\prime}$ region around $\mathrm{HH} 1-2$ (hereafter the $\mathrm{HH}$ 1-2 field). Many point-like sources in the $\mathrm{HH}$ 1-2 region were detected. In addition to the point-like sources, we detected HH 1-2, NGC 1999, and other nebulosities, but the polarimetric studies of these extended sources will be presented elsewhere in the future. 


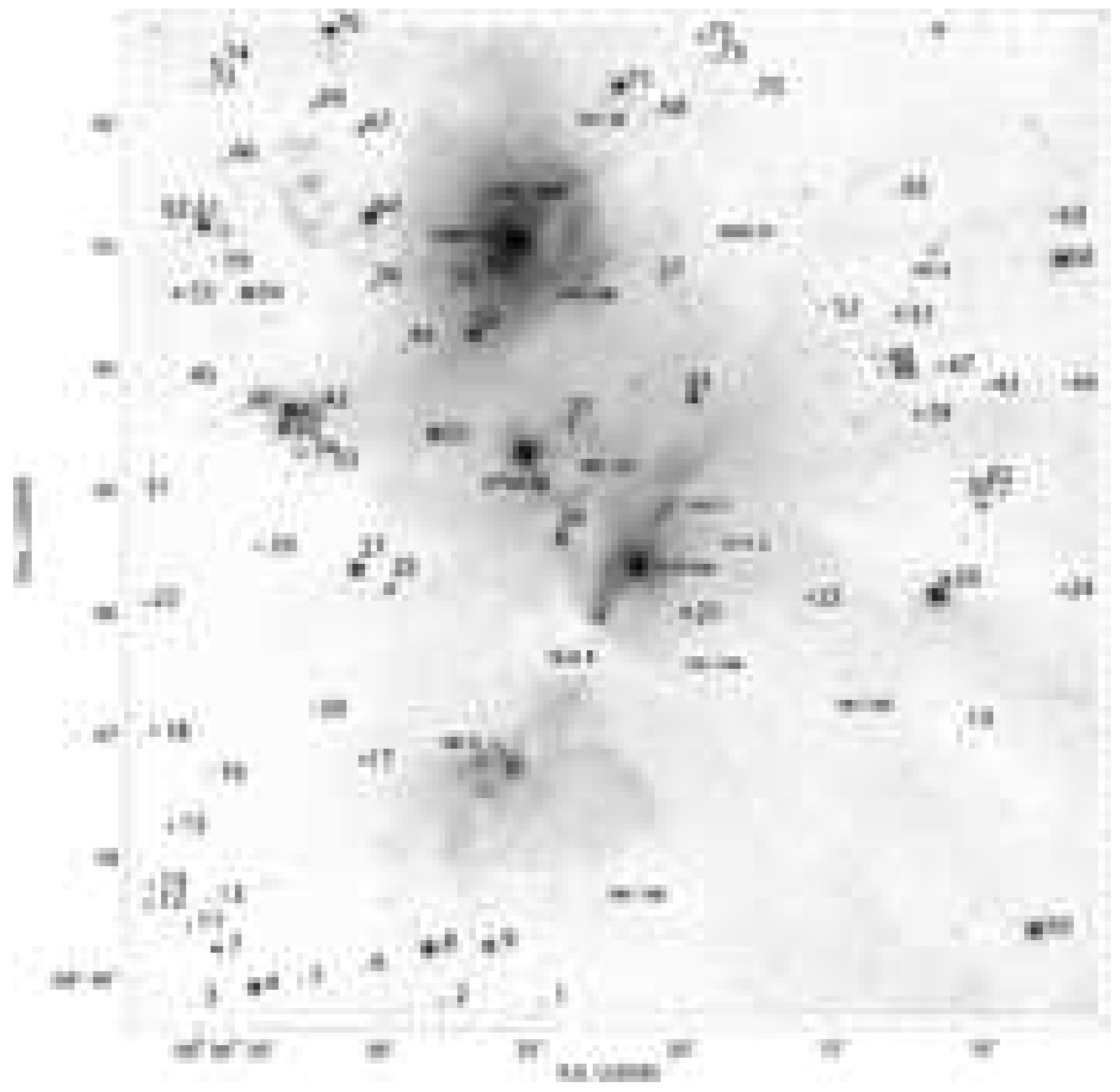

[See http://minho.kasi.re.kr/Publications.html for the original high-quality figure.]

Fig. 2.- Finding chart of the HH 1-2 field (color-negative image of Fig. 1). Detected point-like sources are labeled (Table 1). Bright stars and some extended sources are also labeled.

\section{RESULTS}

\subsection{Photometry}

The IRAF DAOPHOT package was used for source detection and photometry (Stetson 1987). The DAOPHOT program automatically detected point-like sources with peak intensities greater than $10 \sigma$ above the local sky background, where $\sigma$ is the rms uncertainty. The automatic detection procedure misidentified some spurious sources and missed some real sources, and the source list was corrected by visually inspecting the images carefully. The pixel coordinates of the detected sources were matched with the celestial coordinates of their counterparts in the Two Micron All Sky Survey (2MASS) Point Source
Catalog. The IRAF IMCOORDS package was applied to the matched list to obtain plate transform parameters. The rms uncertainty in the coordinate transformation was $\sim 0$.' 1 .

Aperture photometry was performed again with the resulting images. The aperture radius was 3 pixels, and the sky annulus was set to 10 pixels with a 5 pixel width. The resulting list contains 76 sources whose photometric uncertainties are less than $0.1 \mathrm{mag}$ in all three bands (Table 1). These point-like sources are labeled in Figure 2. Four bright sources (V380 Ori, the C-S star, $\mathrm{N}^{3} \mathrm{SK} 50$, and an unnamed star $\sim 10^{\prime \prime}$ south of source 26) were saturated, and they were excluded from the list.

The Stokes $I$ intensity of each point-like source was calcu- 
lated by

$$
I=\frac{1}{2}\left(I_{0}+I_{22.5}+I_{45}+I_{67.5}\right)
$$

where $I_{a}$ is the intensity with the half wave plate oriented at $a^{\circ}$. The magnitude and color of the photometry were transformed into the 2MASS system by

$$
\mathrm{MAG}_{2 \mathrm{MASS}}=\mathrm{MAG}_{\mathrm{IRSF}}+\alpha_{1} \times \mathrm{COLOR}_{\mathrm{IRSF}}+\beta_{1}
$$

and

$$
\mathrm{COLOR}_{2 \mathrm{MASS}}=\alpha_{2} \times \mathrm{COLOR}_{\mathrm{IRSF}}+\beta_{2},
$$

where $M A_{\text {IRSF }}$ is the instrumental magnitude from the IRSF images, and $\mathrm{MAG}_{2 \mathrm{MASS}}$ is the magnitude from the $2 \mathrm{MASS}$ Point Source Catalog. The parameters were determined by fitting the data using a robust least absolute deviation method. For the magnitudes, $\alpha_{1}=0.017,-0.064$, and 0.001 , and $\beta_{1}=$ $-4.986,-4.717$, and -5.375 for $J, H$, and $K_{s}$, respectively. For the colors, $\alpha_{2}=1.007$ and 0.960 , and $\beta_{2}=-0.261$ and 0.664 for $J-H$ and $H-K_{s}$, respectively. The coefficients $\beta_{1}$ and $\beta_{2}$ include both the zero point correction and aperture correction. The derived magnitudes are listed in Table 1 . The $10 \sigma$ limiting magnitudes were $19.6,18.7$, and 17.3 for $J, H$, and $K_{s}$, respectively.

\subsection{Polarimetry}

Aperture polarimetry was carried out on the combined intensity images for each wave plate angle, instead of using the Stokes $Q$ and $U$ images. This is because the center of the sources cannot be determined satisfactorily on the $Q$ and $U$ images. From the aperture photometries on each wave plate angle image, the Stokes parameters of each point-like source were derived by

$$
Q=I_{0}-I_{45}
$$

and

$$
U=I_{22.5}-I_{67.5}
$$

The aperture and sky radius were the same as those used in the photometry of $I$ images. The degree of polarization, $P$, and the polarization position angle, $\theta$, can be calculated by

$$
\begin{aligned}
& P_{0}=\frac{\sqrt{Q^{2}+U^{2}}}{I}, \\
& P=\sqrt{P_{0}^{2}-\delta P^{2}},
\end{aligned}
$$

and

$$
\theta=\frac{1}{2} \arctan \frac{U}{Q},
$$

where $\delta P$ is the uncertainty in $P_{0}$. Equation (7) is necessary to debias the polarization degree (Wardle \& Kronberg 1974). Finally, $P$ was corrected using the polarization efficiencies of SIRPOL: $95.5 \%, 96.3 \%$, and $98.5 \%$ at $J, H$, and $K_{s}$, respectively (Kandori et al. 2006).

Table 2 shows the derived source parameters. The uncertainties given in Table 2 (and elsewhere in this paper) are $1 \sigma$ values. Figures 3-5 show the polarization vector maps of point-like sources superposed on the $I$ images. For the sources with $P / \delta P$ $\geq 4$ and $P<9 \%$ (21 sources), the correlation coefficients are 0.91 for $\left(\theta_{H}, \theta_{K_{s}}\right)$ and 0.97 for $\left(\theta_{H}, \theta_{J}\right)$. Figure 6 shows the histograms and Gaussian fits for the polarization position angles. Each of the three histograms shows a single peak at $\sim 111^{\circ}$.

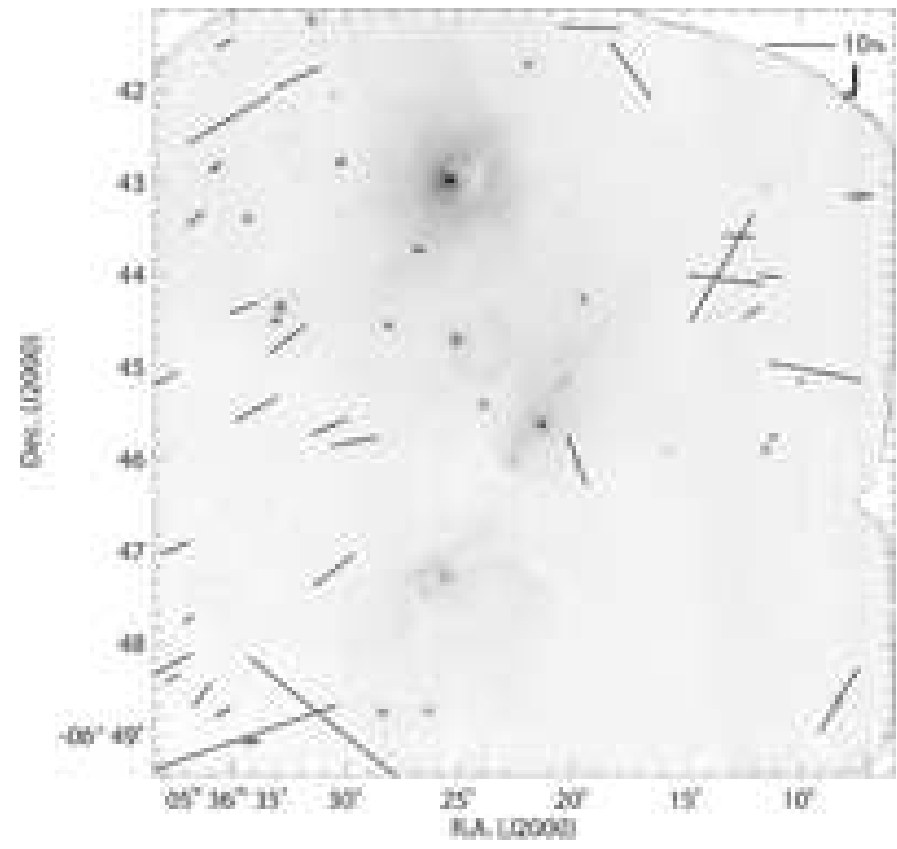

[See http://minho.kasi.re.kr/Publications.html for the original high-quality figure.]

Fig. 3.- Stokes $I$ image of the $J$ band with polarization vectors. The length of the vectors is proportional to the degree of polarization. Shown in the upper right corner is a $10 \%$ vector. Note that there are bad pixel clusters around the upper-left and upper-right corners and the middle of the right boundary.

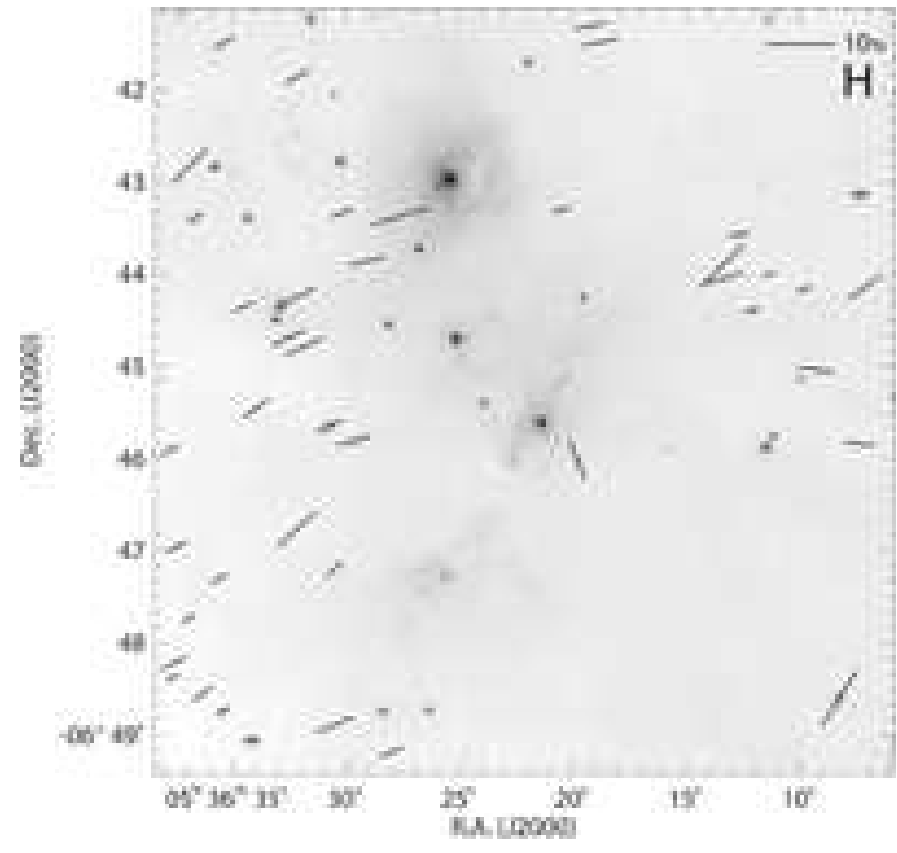

[See http://minho.kasi.re.kr/Publications.html for the original high-quality figure.]

Fig. 4.- The same as Fig. 3 for the $H$ band.

Note that the dispersion in $\theta$ is smallest in the $H$ band. In addition, for most sources, the signal-to-noise ratio $(P / \delta P)$ is higher in the $H$ band than the other bands. The $H$ band polarimetry is more reliable than those of the other bands probably because 


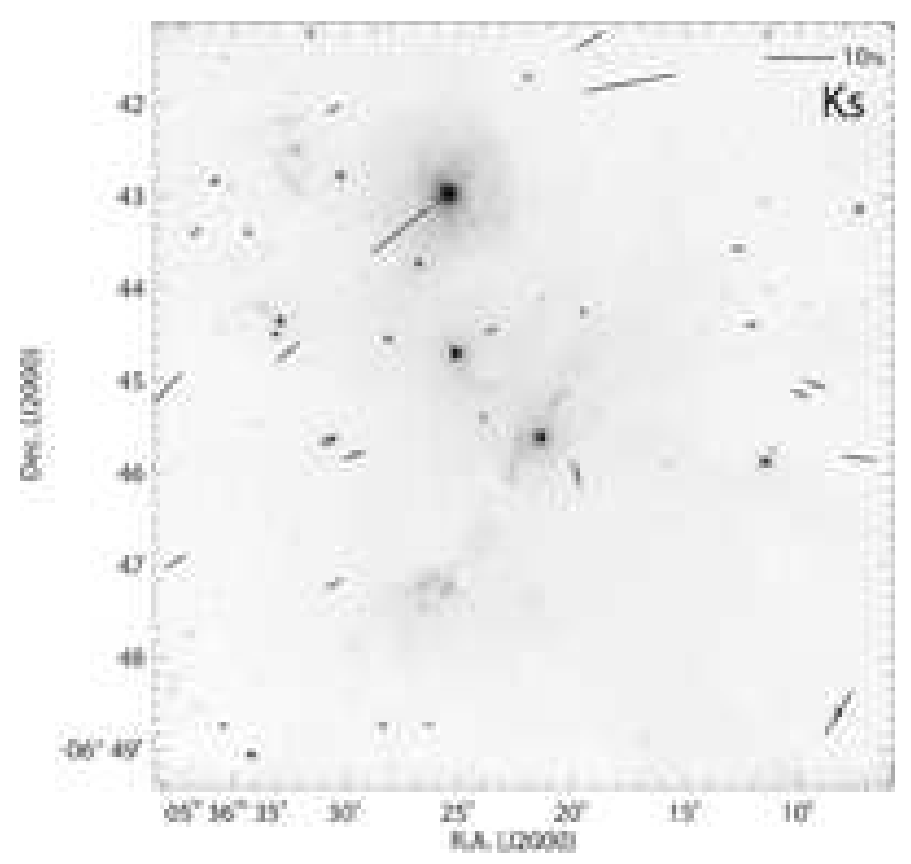

[See http://minho.kasi.re.kr/Publications.html for the original high-quality figure.]

Fig. 5.- The same as Fig. 3 for the $K_{s}$ band.

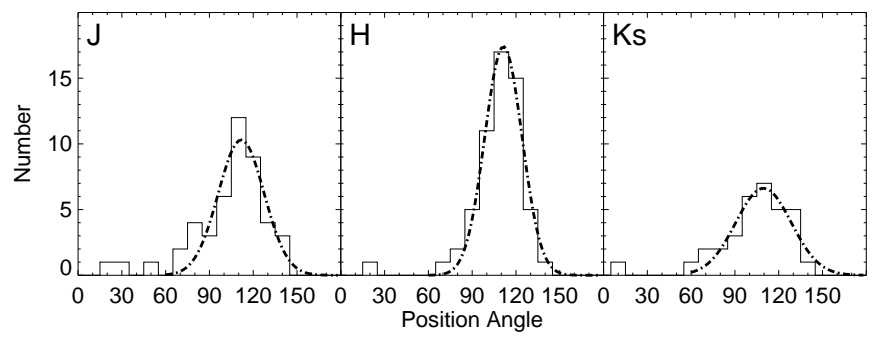

Fig. 6.- Histograms of polarization position angles for the $J$, $H$, and $K_{s}$ bands. All the sources in Table 2 with detected polarization are included. Dot-dashed curves: Gaussian fits. The peak angle and dispersion are $112^{\circ}$ and $16^{\circ}$ for $J, 111^{\circ}$ and $13^{\circ}$ for $H$, and $110^{\circ}$ and $19^{\circ}$ for $K_{s}$.

the contamination from extended nebulosity is smaller in the $H$ band than in the $J$ band and because the dichroic polarization is more efficient in the $H$ band than in the $K_{s}$ band. Therefore, our discussion in Section 4 will be mainly based on the $H$ band data.

The relation between the polarimetric and spectral data may be useful in understanding the nature of polarization. The degree of polarization appears to be correlated with near-IR colors (Fig. 7). The empirical relation for the upper limit of interstellar polarization suggested by Jones (1989) is

$$
P_{K, \max }=\tanh \left\{1.5 E(H-K) \frac{1-\eta}{1+\eta}\right\},
$$

where $\eta=0.875$ and $E(H-K)$ is the reddening owing to extinction. Most of the sources are within this limit (Fig. 7). A few sources are above the $P_{\max }$ limit, but their uncertainties are large. The near-IR polarization-to-extinction efficiency of the point-like sources in the $\mathrm{HH} \mathrm{1-2} \mathrm{field} \mathrm{is} \mathrm{consistent} \mathrm{with} \mathrm{that}$

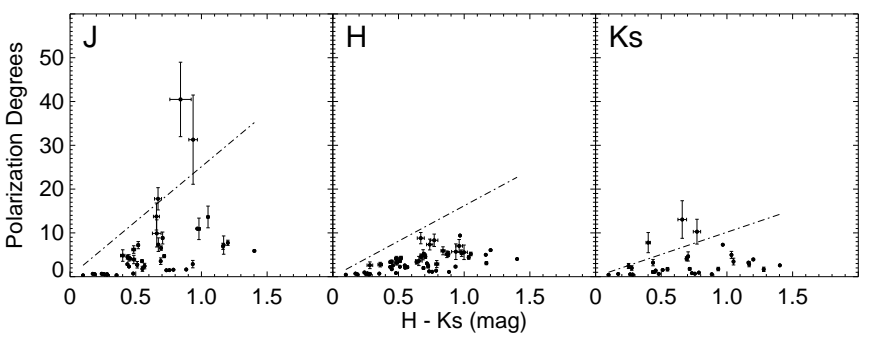

Fig. 7.- Degree of polarization vs. $H-K_{s}$ color. Dot-dashed lines: empirical upper limits ( $P_{\max }$; Jones 1989).

caused by aligned dust grains in the dense interstellar medium. Therefore, their polarizations are likely dominated by the interstellar dichroic extinction, and the intrinsic polarization, if any, did not significantly enhance the degree of polarization. However, this result does not completely exclude the possibility that some of the sources have intrinsic polarization because depolarization is also possible.

\section{DISCUSSION}

\subsection{Comparison with Previous Studies}

Polarimetry of bright point-like sources in the $\mathrm{HH}$ 1-2 region was reported previously by several authors. These studies covered larger regions than our study, but they were much shallower. Strom et al. (1985) carried out $I$ band polarimetry and measured the polarization position angle of two sources: $157^{\circ}$ for the C-S star and $135^{\circ}$ for $\mathrm{N}^{3} \mathrm{SK} 50$. Casali (1995) measured the $K$ band polarization angle of two sources: $105^{\circ}$ for $\mathrm{V} 380$ Ori and $154^{\circ}$ for $\mathrm{N}^{3} \mathrm{SK} 50$. These three sources were saturated in our observations, and no direct comparison is possible.

WS presented a broad-band (450-1000 nm) polarimetry of eight point-like sources in a larger $\left(\sim 10^{\prime}\right)$ region. The polarization position angle averaged about $130^{\circ}$ with a dispersion of $30^{\circ}$. The polarization angle of bright sources were $125^{\circ}$ for the $\mathrm{C}-\mathrm{S}$ star and $128^{\circ}$ for $\mathrm{N}^{3} \mathrm{SK} 50$. Direct comparisons for polarization angles are possible for three sources, while meaningful comparisons for polarization degrees are difficult due to wavelength dependence. WS 1 (source 1 of WS) corresponds to our source 8 , and the polarization position angle of $\mathrm{WS}\left(145^{\circ} \pm 5^{\circ}\right)$ is somewhat larger than our near-IR measurements $\left(95-121^{\circ}\right)$. WS 5 and WS 6 correspond to our sources 10 and 58, respectively, and their polarization orientations agree reasonably well, within $5^{\circ}$. It is not clear what caused the difference of source 8. One of the possibilities is that the polarization of source 8 is caused mostly by the scattering process rather than dichroic extinction, as this source shows little extinction (see Section 4.7 for more discussions).

Based on the broad-band polarimetry, WS suggested that the magnetic field in the $\mathrm{HH} 1-2$ region is oriented at a position angle of $126^{\circ}$. This interpretation, however, should be corroborated by deeper observations because the size of their source sample was too small. Our observations can provide statistically more significant interpretations, which are presented below.

\subsection{Source Classification}

To study the magnetic field structure of molecular clouds through the interstellar polarization caused by dichroic extinction, it is necessary to select sources without intrinsic polarization. YSOs in the cloud can exhibit a substantial degree of 
intrinsic polarization caused by circumstellar material. Such sources may show a large amount of infrared excess emission. Therefore, it is important to classify sources, and the multiwavelength photometry can be useful.

Figure 8 shows a color-color diagram for all the sources detected in all three bands. The diagram was divided into several domains. Based on the location in this diagram, sources can be classified into a few groups (Lada \& Adams 1992). The area near the locus of main-sequence/giant stars is called domain A0. Sources in domain A0 are either field stars (dwarfs and giants) or pre-main-sequence (PMS) stars with little infrared excess (weak-lined T Tauri stars and some classical T Tauri stars) and with little reddening. There is a clear gap just above domain $\mathrm{A} 0$, and the area above this gap in the direction of the reddening vector is called domain Ar. Sources in domain Ar are either field stars or PMS stars with little infrared excess and with substantial reddening. Domain B is the area next to domain Ar in the direction of higher $H-K_{s}$ (to the right) and above the locus of classical T Tauri stars. Sources in domain B are PMS stars with infrared excess emission from disks. Domain $\mathrm{C}$ is the area next to domain $\mathrm{B}$ to the right. Sources in domain $\mathrm{C}$ are infrared protostars or Class I sources. Herbig AeBe stars tend to occupy lower parts of domains B and C. This classification based on the color-color diagram, however, is far from perfect. A certain fraction of classical $\mathrm{T}$ Tauri stars may reside in domains $\mathrm{A} 0$ or $\mathrm{Ar}$, some protostars can be found in domain $\mathrm{B}$, and some extremely reddened AeBe stars may be found among protostars (Lada \& Adams 1992). This "contamination" will even-

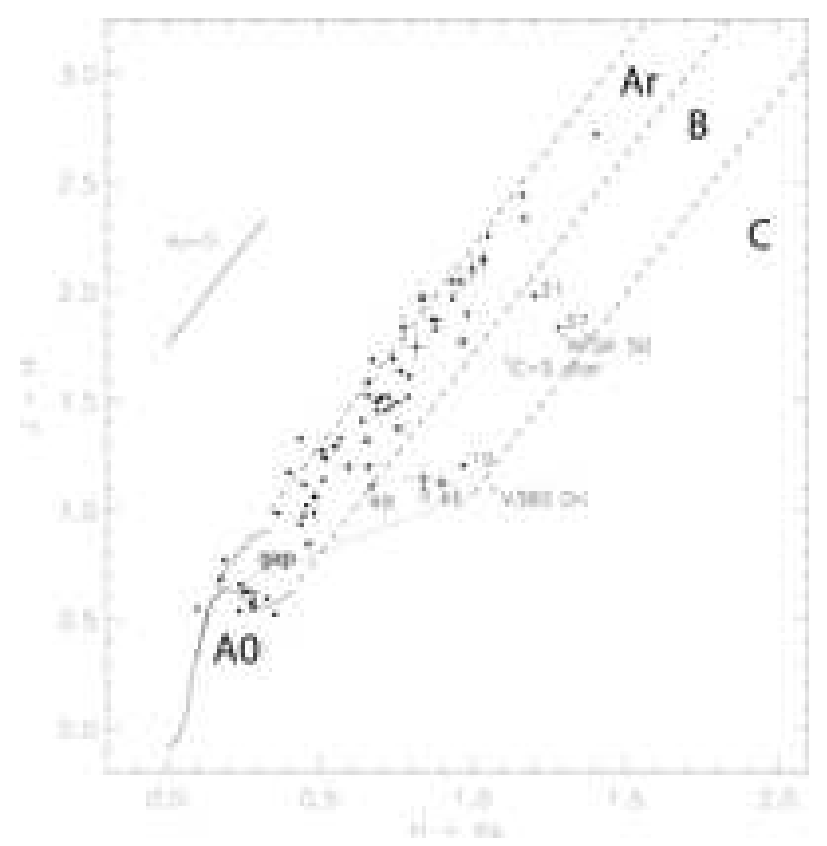

[See http://minho.kasi.re.kr/Publications.html for the original high-quality figure.]

Fig. 8.- Color-color diagram of the point-like sources in the HH 1-2 field. Filled circles: point-like sources from this work (Table 1). Open circles: bright sources (Strom et al. 1989; Carpenter et al. 2001). Solid curve: locus of main sequence and giant branch stars (Bessell \& Brett 1988). Dotted line: locus of classical T Tauri stars (Meyer et al. 1997). Dashed lines: boundaries between domains Ar, B, and C (see Section 4.2). Solid line: reddening vector. tually contribute to the uncertainty in statistical quantities derived from the classification, but the estimation of this uncertainty is beyond the scope of this paper.

There are thirteen sources in domain A0, and they are collectively called group A0. They are either foreground stars or those seen along lines of sight with little extinction.

Fifty seven sources were found in domain Ar. These sources (group Ar) are either background stars or PMS stars in the L1641 cloud. They are the most useful sources for the study of the magnetic fields in the cloud (Section 4.4).

Six sources were found in domain B. These sources (group B) may be PMS stars associated with the L1641 cloud. Source 10 (WS 5) is the emission-line star AY Ori (Wouterloot \& Brand 1992) and also source 13932 of Carpenter et al. (2001). Source 21 is source 14843 of Carpenter et al. (2001). In addition, two of the brightest objects in this field, the C-S star and $\mathrm{N}^{3} \mathrm{SK} 50$, also belong to group $\mathrm{B}$.

None of our sources are located in domain C. However, this nondetection does not mean that there is no protostar in the HH 1-2 field. Some protostars (for example, HH 1-2 VLA 1) are deeply embedded and undetectable in the near-IR bands. In addition, the red protostars tend to be missed at shorter wavelengths ( $J$ and/or $H$ bands). The Herbig Ae star V380 Ori is located in the lower part of domain $\mathrm{C}$, as expected.

\subsection{Source of Polarization}

To study the magnetic field structure of L1641, we will use the distribution of the polarization of group Ar sources (Section 4.4). To do that, the source of polarization needs some discussion. The distribution of polarization angle (Fig. 6) shows a well-defined single peak, which suggests that the cause of polarization is relatively simple. Especially for the group Ar source, the polarization seems to be mostly caused by the L1641 cloud, based on several lines of evidence described below.

First, optically thick molecular lines (such as $\mathrm{CO}$ and $\mathrm{H}_{2} \mathrm{CO}$ ) observed toward the $\mathrm{HH}$ 1-2 region show a single velocity component at $\sim 8 \mathrm{~km} \mathrm{~s}^{-1}$ (Loren et al. 1979; Snell \& Edwards 1982), which suggests that L1641 is the only molecular cloud that is optically thick enough to cause the dichroic extinction. The spectrum of ${ }^{13} \mathrm{CO}$, however, shows that there are two velocity components: a stronger one at $\sim 8.5 \mathrm{~km} \mathrm{~s}^{-1}$ and a weaker one at $\sim 7 \mathrm{~km} \mathrm{~s}^{-1}$ (Edwards \& Snell 1984), and both components belong to the L1641 cloud (Sakamoto et al. 1997). The stronger component is directly related to the dense gas in the $\mathrm{HH}$ 1-2 region, and the weaker component is probably related to the dense gas with a column density peak located $\sim 20^{\prime}$ north of HH 1-2 (Takaba et al. 1986). Since the distribution of the polarization angle shows a well-defined single peak, one of them (most likely the $\sim 8.5 \mathrm{~km} \mathrm{~s}^{-1}$ component) probably dominates in the polarization process. Alternatively, the magnetic field direction of the two components may be very similar.

Second, the peak polarization angle is insensitive to the amount of extinction. If group Ar sources would have a preferred direction of polarization even before their IR photons are affected by the L1641 cloud, sources with a small extinction would show the effect of this "background" polarization direction, while sources with a large extinction would reflect only the polarization caused by L1641. Figure 9 shows the histograms of the polarization position angle for three subgroups of sources grouped by the amount of extinction. All the three subgroups show a peak angle at $\sim 110^{\circ}$. Therefore, the polarization of the group Ar sources are mostly caused by the L1641 cloud only, and the distribution of the polarization angle may be a very good tracer of the magnetic field structure of L1641. 


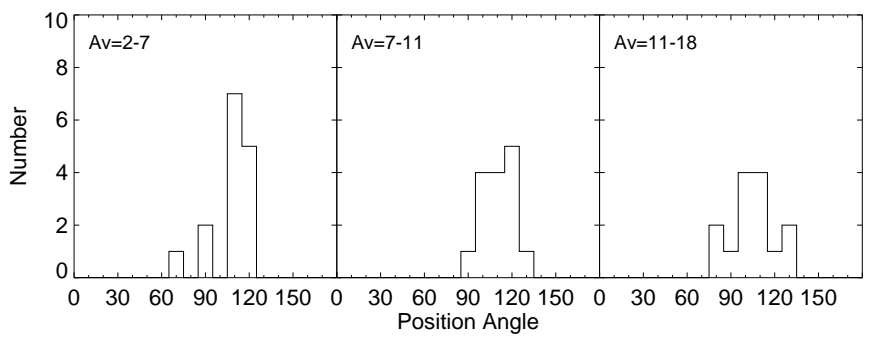

Fig. 9.- Histograms of the $H$ band polarization position angles of group Ar. The sources were grouped by the amount of extinction: $A_{V}=2-7,7-11$, and 11-18. The subgroups contain 15,15 , and 14 sources, respectively.

Third, the Galactic latitude of L1641 is high $(b=-19.8)$. It is unlikely that light from distant luminous giants would suffer a significant amount of dichroic extinction by any other cloud behind L1641.

For some clouds, the polarization direction of background stars can be caused by several sources of polarization along the line of sight. For example, in the direction of the Southern Coalsack dark cloud $\left(b \approx-1^{\circ}\right)$, there are at least three components of polarization (Andersson \& Potter 2005). In such cases, the interpretation of the polarization and its relation with the magnetic field structure can be quite complicated. In the case of the HH 1-2 region, the polarization seems to be mostly caused by L1641, and the relation between the polarization direction and the magnetic field structure is relatively straightforward, as discussed in the next section.

\subsection{Magnetic Field Structure}

The sources in group Ar are best for studying the magnetic field structure of the molecular cloud because they are subject to dichroic extinction and because they would have relatively little intrinsic polarization. Figure 10 shows the histogram and Gaussian fit for the polarization position angles of group Ar sources. The peak angle is $111^{\circ}$, and the dispersion is $11^{\circ}$. Comparing Figures 6 and 10, it is clear that selecting only group Ar sources makes the statistical noise smaller: outliers disappeared, and the dispersion became smaller. Therefore, we suggest that the global magnetic field in the $\mathrm{HH} 1-2$ region is oriented at a position angle of $\sim 111^{\circ}$. This orientation is consistent with the large-scale field structure of L1641 (Vrba et al. 1988). Previous studies of the HH 1-2 region (Strom et al. 1985; WS) suggested larger position angles and larger dispersions because their sample sizes were too small and because they included bright PMS stars in the sample.

To see whether there is a systematic gradient of magnetic field orientation over the imaged field, we divided the HH 12 field into $16(4 \times 4)$ subregions. In each subregion, group Ar sources were selected, and their polarization position angles were averaged. Inspection of the resulting distribution of polarization angle (Fig. 11) did not reveal any systematic trend. Therefore, we suggest that the measured dispersion of $11^{\circ}$ represents the local variation of magnetic field orientation. Here, "local" means each line of sight, though it should be noted that the variation along the line of sight would affect the polarization in an integrative way.

Although the polarization measurement does not provide a direct estimate of the magnetic field strength at each data point in the image, a rough estimation over a large region is pos-

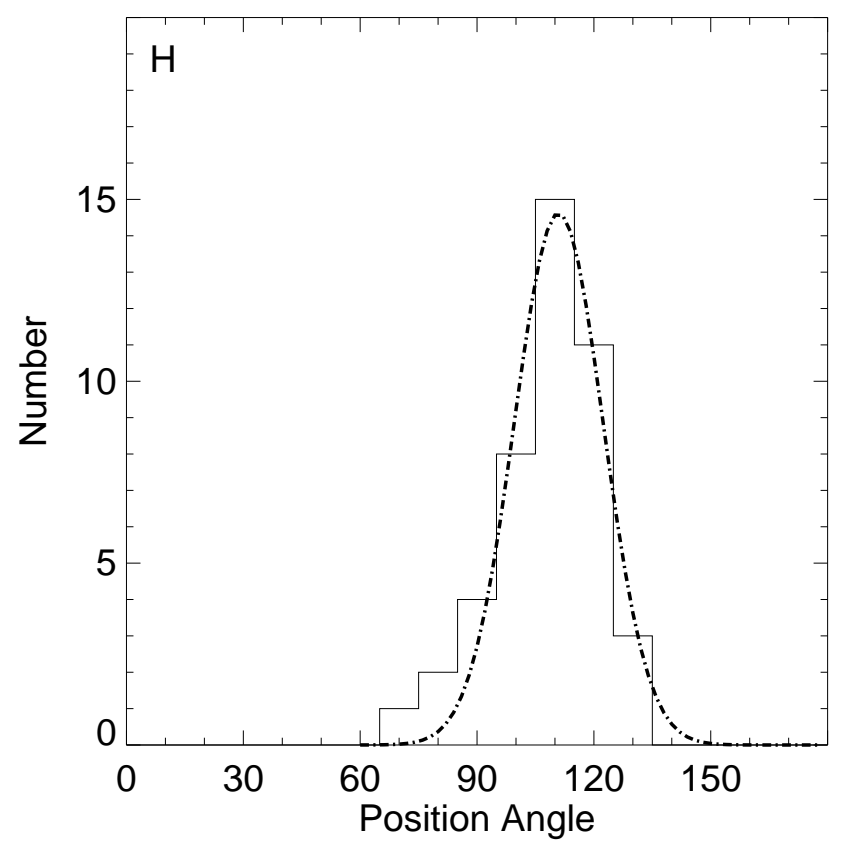

Fig. 10.- Histogram of polarization position angles for the group Ar sources in the $H$ band. Dot-dashed curve: Gaussian fit.

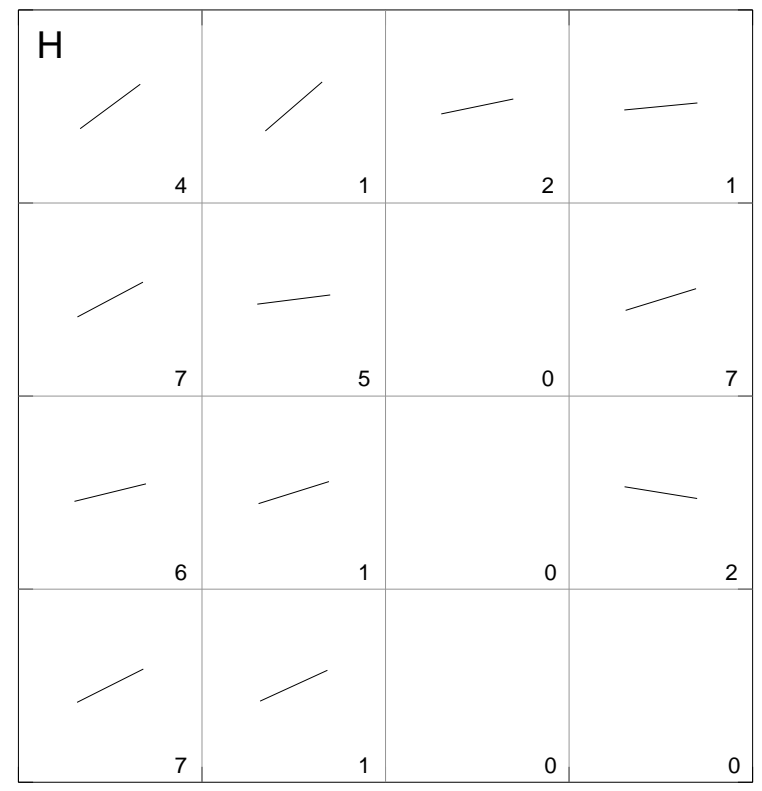

Fig. 11. - Average polarization position angle of group Ar sources in $2^{\prime} \times 2^{\prime}$ subregions of the HH 1-2 field. The number of sources in each subregion is labeled.

sible by statistically comparing the dispersion of polarization orientation with the degree of turbulence in the cloud (Chandrasekhar \& Fermi 1953). Assuming that velocity perturbations are isotropic, the strength of the magnetic field projected on the 
plane of the sky can be calculated by

$$
B_{p}=\mathcal{Q} \sqrt{4 \pi \rho} \frac{\delta v_{\mathrm{los}}}{\delta \theta},
$$

where $\mathcal{Q}$ is a factor to account for various averaging effects, $\rho$ is the mean density of the cloud, $\delta v_{\text {los }}$ is the rms line-of-sight velocity, and $\delta \theta$ is the dispersion of polarization angles. Ostriker et al. (2001) suggested that $\mathcal{Q} \approx 0.5$ is a good approximation when the angle dispersion is small $\left(\delta \theta \lesssim 25^{\circ}\right)$ from numerical simulations. From the observations of the molecular condensation in the $\mathrm{CO}$ and ${ }^{13} \mathrm{CO} J=1 \rightarrow 0$ lines, Takaba et al. (1986) estimated an $\mathrm{H}_{2}$ column density of $2.5 \times 10^{22} \mathrm{~cm}^{-2}$ and a size of $2.0 \mathrm{pc}$. The density $\rho$ can be derived by assuming that the line-of-sight size of the dense condensation is similar to the lateral size. The FWHM line width of the $\mathrm{C}^{18} \mathrm{O} J=1$ $\rightarrow 0$ line, $2.7 \mathrm{~km} \mathrm{~s}^{-1}$ (Takaba et al. 1986), can be used to estimate $\delta v_{\text {los }}$. Then the derived field strength is $B_{p} \approx 130 \mu \mathrm{G}$. The uncertainty in this estimate may be rather large because the observed HH 1-2 field is only a part of the L1641 cloud, and it should be taken as an order-of-magnitude estimate. The estimated magnetic field strength of the $\mathrm{HH} 1-2$ region is similar to that of other molecular clouds $(20-200 \mu \mathrm{G})$ derived using the Chandrasekhar-Fermi method (e.g., Andersson \& Potter 2005; Poidevin \& Bastien 2006; Alves et al. 2008).

An interesting issue is how good near-IR polarimetry is in tracing the magnetic field structure of dense clouds. Goodman et al. (1995) suggested that the polarizing power of dust grains may drop in the dense interior of some dark clouds and that near-IR polarization maps of background sources may be unreliable. However, the relevant physics is surprisingly complex (Lazarian 2007), and there are observational evidence and theoretical explanations for aligned grains in dense cloud cores (Ward-Thompson et al. 2000; Cho \& Lazarian 2005). In the case of our study of the $\mathrm{HH}$ 1-2 field, the polarization degree $P_{H}$ does not show a clear sign of saturation up to $H-K_{s} \approx 1$ (Fig. 7), and the observed polarizations of detected sources may well represent the magnetic fields in the $\mathrm{HH} 1-2$ region.

\subsection{Pre-Main-Sequence Stars with Infrared Excess}

Sources in group B are PMS stars with infrared excess. In the study of global magnetic fields, we excluded group B sources because they may have intrinsic polarizations. To verify this precaution, their polarization properties may be compared with those of group Ar.

Figure 12 shows the histogram of the polarization position angles of group B sources from this work and three bright PMS stars from the literature. The distribution is relatively widespread, and the peak angle is ill-defined. Probably it is not

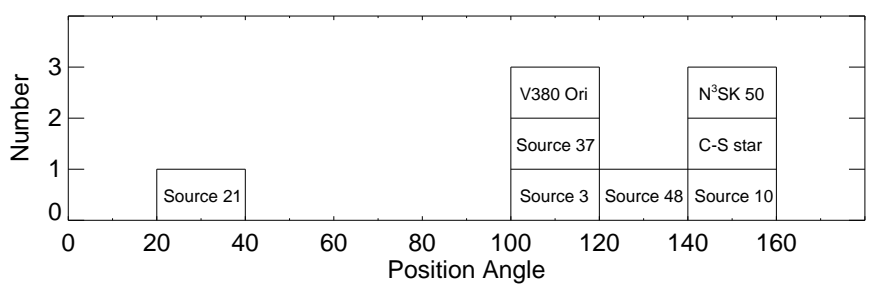

Fig. 12.- Histogram of near-IR polarization position angles for the group B sources and the bright PMS stars (Strom et al. 1985; Casali 1995). Note that a $20^{\circ}$ bin is used because the sample size is small. a single-peaked distribution (cf. Tamura \& Sato 1989), but the sample size is too small to discuss in detail. Quantifying the dispersion is difficult, but it is clearly much larger than that of group Ar. This distribution implies that these PMS stars have significant intrinsic polarizations and that the orientation of the intrinsic polarization has no strong correlation with that of the global magnetic fields.

\subsection{Outflow Orientations}

The alignment between the magnetic fields of a star-forming cloud and the orientation of resulting stars is an interesting topic, because it can provide information on the role of magnetic fields during the protostellar collapse and subsequent evolution of the system. In an ideal scenario of quasi-static isolated single star formation, one would expect that the symmetry axis of the newly formed (proto)stellar system (such as the rotation axis, bipolar outflow axis) would be parallel to the magnetic field that threaded the initial dense cloud core (e.g., Galli \& Shu 1993), which would imply that the distribution of outflow orientations would be similar to that of the polarization angle of background sources (with a correction for the averaging effects, see below). Strom et al. (1986) found that about $70 \%$ of the optical flows in their imaging survey have the outflow directions within $30^{\circ}$ of the magnetic field direction of the cloud they belong to. However, in a recent survey of classical T Tauri stars, Ménard \& Duchêne (2004) found that the jets/disks around T Tauri stars are oriented randomly with respect to the cloud magnetic fields.

In comparing the distributions of the polarization angle and the outflow direction, the dispersion of the polarization angle should be corrected by a certain factor, because the averaging effects along the line of sight would decrease the dispersion. While it is impossible to find an exact correction factor for each cloud, numerical simulations suggests that it is in the range of 0.46-0.51 when the dispersion is small (Ostriker et al. 2001). Andersson \& Potter (2005) tackled this problem using Monte Carlo simulations to analyze the polarimetry of the Southern Coalsack cloud and found that a reasonable correction factor would be in the range 0.3-1.0. This factor tends to be large (close to 1) when the cloud is nearly homogeneous and can be small when there are many distinct regions along the line of sight. The HH 1-2 region is not completely homogeneous, as the molecular cloud has two velocity components along the line of sight (see Section 4.3), and also not as complicated as the Southern Coalsack cloud. Therefore, a correction factor of $\sim 0.5$ would be a reasonable value.

For the HH 1-2 region, previously much attention was paid to the relation between the direction of the $\mathrm{HH} 1-2$ outflow (position angle $\approx 148^{\circ}$ ) and the orientation of global magnetic fields (Strom et al. 1985; WS). Our polarimetry shows that the difference between them $\left(\sim 40^{\circ}\right)$ is much larger than the $11^{\circ}$ dispersion of the polarization angles. Therefore, there is a certain degree of misalignment. To make a more meaningful comparison, a list of optical jets in the HH 1-2 field with known flow directions was compiled (Table 3). The driving sources of these outflows are YSOs. Figure 13 shows the distribution of the outflow orientations. Considering the distribution of the polarization angle and the correction factor mentioned above, the expected distribution of the outflow direction would show a single peak at $\sim 110^{\circ}$ with a dispersion of $\sim 20^{\circ}$. Surprisingly, the position angle of the outflow shown in Figure 13 seems to be nearly random, i.e., the distribution is almost uniform, which makes a stark contrast to the distribution of polarization angles. Therefore, we suggest that many protostars may become disori- 


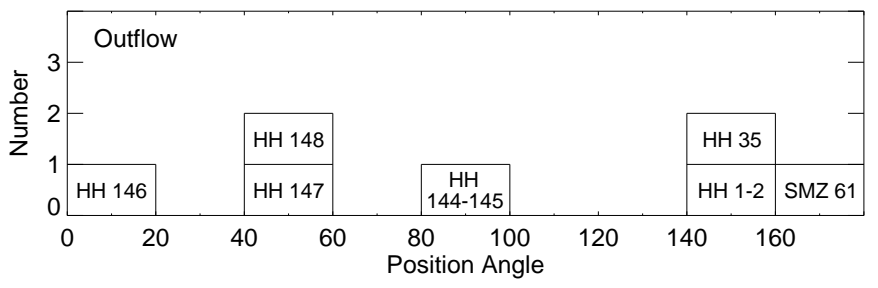

Fig. 13. - Histogram of outflow orientations of optical jets in the HH 1-2 region.

ented during the star forming process.

The random orientation of YSOs suggests that the global magnetic field may lose control of a collapsing protostellar core at a certain small scale or at a certain stage of evolution. Several mechanisms may be working to cause the disorientation. First, the dynamical interaction during a binary (or multiple star) formation process can produce a misaligned system (e.g., Bonnell et al. 1992). In fact, misaligned binaries in the Class II phase are not unusual (Monin et al. 2007). Among the outflow driving sources in the HH 1-2 region, V380 Ori and HH 1-2 VLA $1 / 2$ are examples to the point. V380 Ori is a $0{ }^{\prime \prime} 15$ ( $\left.\sim 60 \mathrm{AU}\right)$ binary and drives HH 35 and HH 148, and these two outflows are almost perpendicular to each other (Strom et al. 1986; Leinert et al. 1997). HH 1-2 VLA $1 / 2$ is a $3^{\prime \prime}(\sim 1200$ AU) binary and drives HH 1-2 and HH 144-145, and the projected angle between these flows is $\sim 70^{\circ}$ (Reipurth et al. 1993). Second, even in a single-star formation, an outflow may not be aligned with the magnetic field of the surrounding cloud if the magnetic field is too weak (Matsumoto et al. 2006). There are other possible mechanisms (Ménard \& Duchêne 2004).

Considering that there are Class 0 sources showing misalignments, the disorientation may happen very early. For example, HH 1-2 VLA 1 is a Class 0 source (Chini et al. 1997). Examples in other star-forming regions include HH 24 MMS, which seems to have two accretion disks with a $\sim 45^{\circ}$ difference in projected orientation (Kang et al. 2008), and NGC 1333 IRAS 2 , which seems to drive two outflows almost perpendicular to each other (Hodapp \& Ladd 1995).

\subsection{Wavelength Dependence of Interstellar Polarization}

Though the selected point-like sources have no detectable nebulosity, we cannot rule out the possibility of the presence of unresolved reflection nebulae in some of the sources. Indeed, Casali (1995) showed that the polarization by scattering is important in some of the sources in L1641, especially the sources with low extinction. To discriminate between the contributions from the dichroic extinction and from the scattering, the wavelength dependence of polarization can be measured by calculating the ratio of polarization degrees. In the infrared wavelength range, the polarization by dichroic extinction decreases with wavelength while the polarization by scattering is not a strong function of wavelength (Whittet et al. 1992; Casali 1995).

Figure 14 shows the $P_{J} / P_{K_{s}}$ and $P_{H} / P_{K_{s}}$ ratios for the sources in the HH 1-2 field. It is very clear that groups A0 and $\mathrm{Ar}$ show very different behavior. Within group $\mathrm{Ar}$, the ratios are reasonably constant, and the weighted average values are $P_{J} / P_{K_{s}}=2.18$ and $P_{H} / P_{K_{s}}=1.51$ with standard deviations of 0.10 and 0.07 , respectively. These values are consistent with those for the whole L1641 area (2.5 and 1.4 with $2 \sigma$ scatters of 0.7 and 0.2, respectively; Casali 1995), which is also consistent

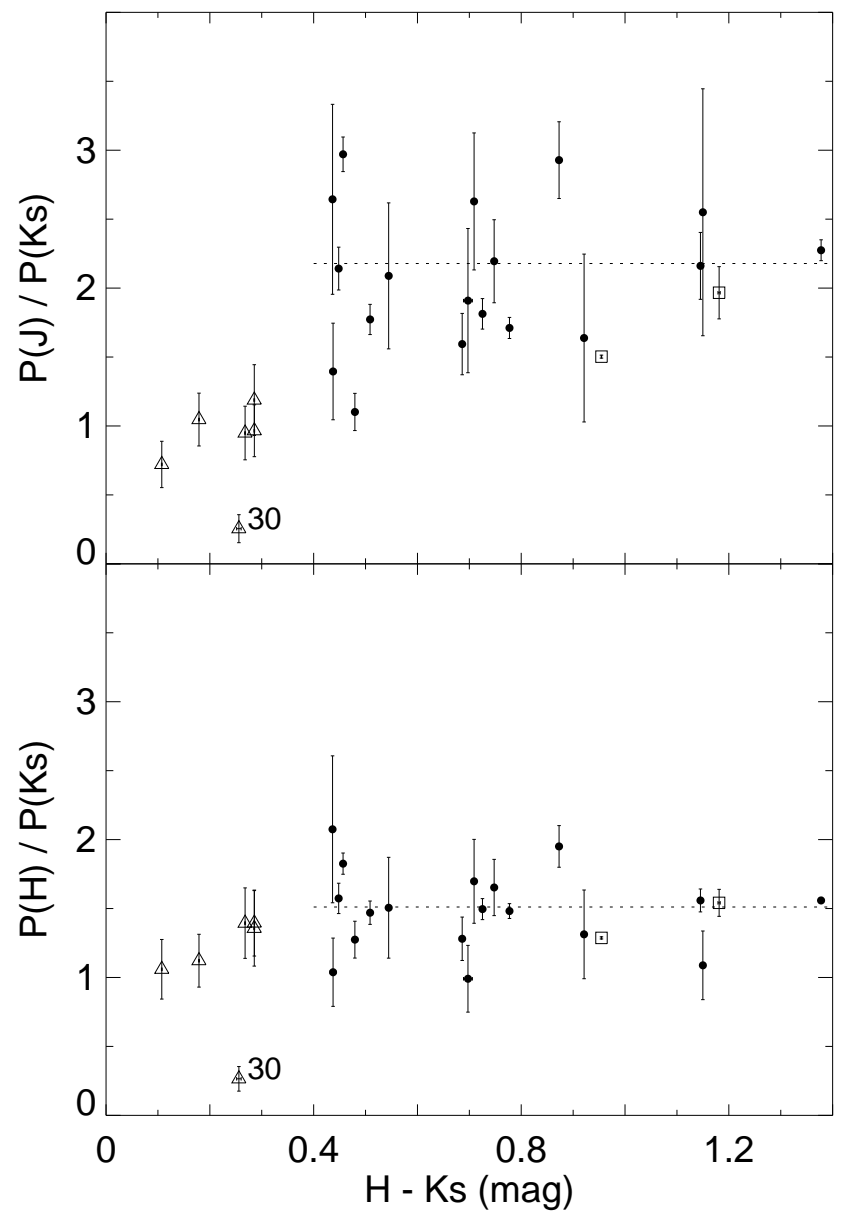

Fig. 14.- The ratio of $J$ to $K_{s}$ polarizations (top panel) and the ratio of $H$ to $K_{s}$ polarizations (bottom panel) against the $H-K_{s}$ color for the 26 sources detected in the $J, H$, and $K_{s}$ bands. Triangles: sources in group A0. Source 30 has unusually low ratios. Filled circles: sources in group Ar. Squares: sources in group B. Dotted lines: weighted average for group Ar.

with the empirical relation $P \propto \lambda^{-\beta}$ with $\beta=1.6-2.0$ (Whittet 1992). Therefore, the polarization of group Ar sources can be very well explained by dichroic extinction.

In contrast, the $P_{J} / P_{K_{s}}$ and $P_{H} / P_{K_{s}}$ ratios of group A0 sources are near unity, which is significantly different from those of group Ar sources. Therefore, the polarization mechanism for low extinction sources may be dominated by the circumstellar scattering (see more discussions in Section 5.4 of Casali 1995). Source 30 shows unusually low ratios (Fig. 14) because its $K_{s}$ band polarization degree is high while the $P_{J} / P_{H}$ ratio is near one as expected. Its $K_{s}$ band polarization angle is also quite different from those of $J$ and $H$ bands. It is not clear what caused this peculiar behavior.

\section{SUMMARY}

We conducted a deep and wide-field $J-H-K_{s}$ imaging polarimetry toward a $8^{\prime} \times 8^{\prime}$ region around $\mathrm{HH} 1-2$ in the starforming cloud L1641. The main results in this study are sum- 
marized as follows.

1. Aperture photometry of point-like sources in the HH 12 field was made. The number of sources detected in all three bands is 76 . These sources were classified using a color-color diagram. There are 57 sources in group Ar, reddened sources with little infrared excess.

2. Aperture polarimetry of the point-like sources resulted in the positive detection of 63 sources in at least one of the three bands. Most of the near-IR polarizations of the point-like sources can be explained by dichroic polarization.

3. Toward the HH 1-2 region, L1641 is the only molecular cloud that is optically thick enough to cause the dichroic extinction. For the group Ar sources, the polarization direction does not depend on the amount of extinction, which suggests that the L1641 cloud is the only source of systematic polarization.

4. Sources in group Ar are expected to be either background stars or PMS stars with little intrinsic polarization. The histogram of polarization position angles of group Ar sources has a well-defined peak at $\sim 111^{\circ}$, which we interpret as the projected direction of the magnetic fields in the HH 1-2 region. From the $11^{\circ}$ dispersion of the polarization angles, a rough estimate of the strength of the magnetic field projected on the plane of the sky is $\sim 130$ $\mu \mathrm{G}$.

5. The orientation of the YSO outflows/jets in the HH 1-2 region appears to be almost random, which is completely different from the distribution of the magnetic field directions in the cloud. This difference suggests that protostars may be disoriented during the star formation process, probably because of the dynamical interaction in multiple systems.

6. For the group Ar sources, the wavelength dependence of polarization is consistent with the dichroic extinction. Sources in group A0 have a small amount of extinction, and their polarization seems to be caused by the circumstellar scattering.

This work was supported by the Korea Science and Engineering Foundation (KOSEF) grant No. 2009-0063616, funded by the Korean government (MEST). M.T. and T.N. are supported by a Grant-in-Aid from the Ministry of Education, Culture, Sports, Science and Technology (No. 19204018). This paper uses observations made at the South African Astronomical Observatory. IRAF is distributed by the US National Optical Astronomy Observatories, which are operated by the Association of Universities for Research in Astronomy, Inc., under cooperative agreement with the National Science Foundation. This publication makes use of data products from the Two Micron All Sky Survey, which is a joint project of the University of Massachusetts and the Infrared Processing and Analysis Center/California Institute of Technology, funded by the National Aeronautics and Space Administration and the National Science Foundation.

\section{REFERENCES}

Alves, F. O., Franco, G. A. P., \& Girart, J. M. 2008, A\&A, 486, L13 Andersson, B.-G., \& Potter, S. B. 2005, MNRAS, 356, 1088

Bergin, E. A., \& Tafalla, M. 2007, ARA\&A, 45, 339

Bessell, M. S., \& Brett, J. M. 1988, PASP, 100, 1134

Bonnell, I., Arcoragi, J.-P., Martel, H., \& Bastien, P. 1992, ApJ, 400, 579

Carpenter, J. M., Hillenbrand, L. A., \& Skrutskie, M. F. 2001, AJ, 121, 3160

Casali, M. M. 1995, MNRAS, 277, 1385

Chandrasekhar, S., \& Fermi, E. 1953, ApJ, 118, 113

Chini, R., Reipurth, B., Sievers, A., Ward-Thompson, D., Haslam, C. G. T., Kreysa, E., \& Lemke, R. 1997, A\&A, 325, 542

Cho, J., \& Lazarian, A. 2005, ApJ, 631, 361

Choi, M., \& Zhou S. 1997, ApJ, 477, 754

Corcoran, D., \& Ray, T. P. 1995, A\&A, 301, 729

Davis, L. J., \& Greenstein, J. L. 1951, ApJ, 114, 206

Edwards, S., \& Snell, R. L. 1984, ApJ, 281, 237

Eislöffel, J., Mundt, R., \& Bohm, K. H. 1994, AJ, 108, 1042

Galli, D., \& Shu, F. H. 1993, ApJ, 417, 243

Goodman, A. A., Jones, T. J., Lada, E. A., \& Myers, P. C. 1995, ApJ, 448,748

Haro, G. 1952, ApJ, 115, 572

Herbig, G. H. 1951, ApJ, 113, 697

Hodapp, K.-W., \& Ladd, E. F. 1995, ApJ, 453, 715

Jones, T. J. 1989, ApJ, 346, 728

Kandori, R., et al. 2006, Proc. SPIE, 6269, 159

Kandori, R., et al. 2007, PASJ, 59, 487

Kang, M., Choi, M., Ho, P. T. P., \& Lee, Y. 2008, ApJ, 683, 267

Kutner, M. L., Tucker, K. D., Chin, G., \& Thaddeus, P. 1977, ApJ, 215, 521

Lada, C. J., \& Adams, F. C. 1992, ApJ, 393, 278

Lazarian, A. 2007, J. Quant. Spectrosc. Radiat. Transfer, 106, 225

Leinert, C., Richichi, A., \& Haas, M. 1997, A\&A, 318, 472

Loren, R. B., Evans, N. J., II, \& Knapp, G. R. 1979, ApJ, 234, 932

Mac Low, M.-M., \& Klessen, R. S. 2004, Rev. Mod. Phys., 76, 125

Maddalena, R. J., Morris, M., Moscowitz, J., \& Thaddeus, P. 1986, ApJ, 303, 375

Matsumoto, T., Nakazato, T., \& Tomisaka, K. 2006, ApJ, 637, L105

Matthews, B. C., \& Wilson, C. D. 2000, ApJ, 531, 868

McKee, C. F., \& Ostriker, E. C. 2007, ARA\&A, 45, 565

Ménard, F., \& Duchêne, G. 2004, A\&A, 425, 973

Meyer, M. R., Calvet, N., \& Hillenbrand, L. A. 1997, AJ, 114, 288

Monin, J.-L., Clarke, C. J., Prato, L., \& McCabe, C. 2007, in Protostars and Planets V, ed. B. Reipurth, D. Jewitt, \& K. Keil (Tucson: Univ. Arizona Press), 395

Morgan, J. A., \& Bally, J. 1991, ApJ, 372, 505

Nagayama, T., et al. 2003, Proc. SPIE, 4841, 459

Ostriker, E. C., Stone, J. M., \& Gammie, C. F. 2001, ApJ, 546, 980

Poidevin, F., \& Bastien, P. 2006, ApJ, 650, 945

Pravdo, S. H., Rodríguez, L. F., Curiel, S., Cantó, J., Torrelles, J. M., Becker, R. H., \& Sellgren, K. 1985, ApJ, 293, L35

Reipurth, B., Heathcote, S., Roth, M., Noriega-Crespo, A., \& Raga, A. C. 1993, ApJ, 408, L49

Rodríguez, L. F., Delgado-Arellano, V. G., Gómez, Y., Reipurth, B., Torrelles, J. M., Noriega-Crespo, A., Raga, A. C., \& Cantó, J. 2000, AJ, 119, 882

Sakamoto, S., Hasegawa, T., Hayashi, M., Morino, J.-I., \& Sato, K. 1997, AJ, 481, 302

Shu, F. H., Adams, F. C., \& Lizano, S. 1987, ARA\&A, 25, 23

Snell, R. L., \& Edwards, S. 1982, ApJ, 259, 668 
Stanke, T., McCaughrean, M. J., \& Zinnecker, H. 2002, A\&A, 392, 239

Stetson, P. B. 1987, PASP, 99, 191

Strom, K. M., Newton, G., Strom, S. E., Seaman, R. L., Carrasco, L., Cruz-Gonzalez, I., Serrano, A., \& Grasdalen, G. L. 1989, ApJS, 71, 183

Strom, K. M., Strom, S. E., Wolff, S. C., Morgan, J., \& Wenz, M. 1986, ApJS, 62, 39

Strom, S. E., Strom, K. M., Grasdalen, G. L., Sellgren, K., Wolff, S., Morgan, J., Stocke, J., \& Mundt, R. 1985, AJ, 90, 2281

Takaba, H., Fukui, Y., Fujimoto, Y., Sugitani, K., Ogawa, H., \& Kawabata, K. 1986, A\&A, 166, 276

Tamura, M., Nagata, T., Sato, S., \& Tanaka, M. 1987, MNRAS, 224, 413

Tamura, M., \& Sato, S. 1989, AJ, 98, 1368

Vrba, F. J., Strom, S. E., \& Strom, K. M. 1976, AJ, 81, 958

Vrba, F. J., Strom, S. E., \& Strom, K. M. 1988, AJ, 96, 680

Wardle, J. F. C., \& Kronberg, P. P. 1974, ApJ, 194, 249

Ward-Thompson, D., Kirk, J. M., Crutcher, R. M., Greaves, J. S., Holland, W. S., \& André, P. 2000, ApJ, 537, L135

Warren-Smith, R. F., \& Scarrott, S. M. 1999, MNRAS, 305, 875 (WS)

Warren-Smith, R. F., Scarrott, S. M., King, D. J., Taylor, K. N. R., Bingham, R. G., \& Murdin, P. 1980, MNRAS, 192, 339

Whittet, D. C. B. 1992, Dust in the Galactic Environment (Bristol: IOP)

Whittet, D. C. B., Martin, P. G., Hough, J. H., Rouse, M. F., Bailey, J. A., \& Axon, D. J. 1992, ApJ, 386, 562

Wilking, B. A., Lebofsky, M. J., Kemp, J. C., \& Rieke, G. H. 1979, AJ, 84, 199

Wouterloot, J. G. A., \& Brand, J. 1992, A\&A, 265, 144

Zavagno, A., Molinari, S., Tommasi, E., Saraceno, P., \& Griffin, M. 1997, A\&A, 325, 685 
TABLE 1

Photometry of PoINT-Like Sources IN THE HH 1-2 Field

\begin{tabular}{|c|c|c|c|c|c|c|}
\hline \multirow[t]{2}{*}{ Source } & \multicolumn{2}{|c|}{ Position } & \multirow{2}{*}{$\begin{array}{c}J \\
(\mathrm{mag})\end{array}$} & \multirow{2}{*}{$\begin{array}{c}H \\
(\mathrm{mag})\end{array}$} & \multirow{2}{*}{$\begin{array}{c}K_{s} \\
(\mathrm{mag})\end{array}$} & \multirow[t]{2}{*}{ 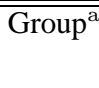 } \\
\hline & $\alpha_{\mathrm{J} 2000.0}$ & $\delta_{\mathrm{J} 2000.0}$ & & & & \\
\hline 1 & 53624.59 & -64911.6 & 17.86 & 16.67 & 16.07 & $\mathrm{Ar}$ \\
\hline 2 & 53627.99 & -64911.1 & 17.32 & 15.99 & 15.34 & $\mathrm{Ar}$ \\
\hline 3 & 53636.22 & -64910.5 & 18.62 & 17.46 & 16.63 & B \\
\hline 4 & 53634.15 & -64903.5 & 13.37 & 12.24 & 11.73 & $\mathrm{Ar}$ \\
\hline 5 & 53632.60 & -64900.1 & 18.08 & 16.81 & 16.30 & $\mathrm{Ar}$ \\
\hline 6 & 53630.50 & -64853.5 & 19.29 & 17.21 & 16.28 & $\mathrm{Ar}$ \\
\hline 7 & 53635.41 & -64844.6 & 14.77 & 13.85 & 13.41 & $\mathrm{Ar}$ \\
\hline 8 & 53628.37 & -64844.5 & 12.65 & 12.15 & 12.04 & A0 \\
\hline 9 & 53626.35 & -64843.4 & 13.58 & 13.09 & 12.73 & A0 \\
\hline 10 & 53608.29 & -64836.2 & 13.18 & 11.95 & 11.00 & B \\
\hline 11 & 53636.32 & -64833.3 & 16.59 & 15.62 & 15.17 & $\mathrm{Ar}$ \\
\hline 12 & 53637.65 & -64822.5 & 16.29 & 14.96 & 14.40 & $\mathrm{Ar}$ \\
\hline 13 & 53635.62 & -64820.1 & 18.61 & 17.22 & 16.47 & $\mathrm{Ar}$ \\
\hline 14 & 53637.57 & -64813.2 & 16.74 & 15.68 & 15.20 & $\mathrm{Ar}$ \\
\hline 15 & 53636.92 & -64744.4 & 16.26 & 14.96 & 14.41 & $\mathrm{Ar}$ \\
\hline 16 & 53635.58 & -64718.4 & 18.09 & 16.46 & 15.67 & $\mathrm{Ar}$ \\
\hline 17 & 53630.54 & -64712.3 & 17.80 & 15.41 & 14.26 & $\mathrm{Ar}$ \\
\hline 18 & 53637.46 & -64657.5 & 16.10 & 14.78 & 14.34 & $\mathrm{Ar}$ \\
\hline 19 & 53610.68 & -64654.3 & 19.44 & 17.67 & 16.86 & $\mathrm{Ar}$ \\
\hline 20 & 53632.15 & -64646.0 & 18.44 & 16.73 & 16.00 & $\mathrm{Ar}$ \\
\hline 21 & 53619.80 & -64600.7 & 16.44 & 14.41 & 13.23 & B \\
\hline 22 & 53637.73 & -64554.2 & 16.66 & 15.69 & 15.33 & $\mathrm{Ar}$ \\
\hline 23 & 53615.70 & -64553.1 & 15.79 & 15.28 & 15.04 & A0 \\
\hline 24 & 53607.34 & -64550.0 & 17.65 & 15.47 & 14.45 & $\mathrm{Ar}$ \\
\hline 25 & 53629.62 & -64548.2 & 16.51 & 14.02 & 12.87 & $\mathrm{Ar}$ \\
\hline 26 & 53611.19 & -64544.5 & 14.51 & 13.88 & 13.63 & A0 \\
\hline 27 & 53630.71 & -64538.5 & 14.93 & 12.14 & 10.76 & $\mathrm{Ar}$ \\
\hline 28 & 53633.95 & -64527.5 & 17.46 & 15.76 & 15.09 & $\mathrm{Ar}$ \\
\hline 29 & 53623.95 & -64523.8 & 13.16 & 12.56 & 12.29 & A0 \\
\hline 30 & 53609.96 & -64508.1 & 15.12 & 14.52 & 14.26 & A0 \\
\hline 31 & 53638.06 & -64508.4 & 16.87 & 15.71 & 15.31 & $\mathrm{Ar}$ \\
\hline 32 & 53609.32 & -64502.0 & 17.94 & 15.64 & 14.61 & $\mathrm{Ar}$ \\
\hline 33 & 53631.84 & -64447.2 & 18.37 & 16.37 & 15.54 & $\mathrm{Ar}$ \\
\hline 34 & 53632.56 & -64441.7 & 16.28 & 14.77 & 14.08 & $\mathrm{Ar}$ \\
\hline 35 & 53628.10 & -64432.5 & 13.20 & 12.22 & 11.74 & $\mathrm{Ar}$ \\
\hline 36 & 53633.07 & -64429.4 & 13.93 & 12.43 & 11.68 & $\mathrm{Ar}$ \\
\hline 37 & 53623.58 & -64427.0 & 17.17 & 15.28 & 14.03 & B \\
\hline 38 & 53612.10 & -64423.3 & 16.78 & 14.77 & 13.85 & $\mathrm{Ar}$ \\
\hline 39 & 53634.49 & -64421.4 & 17.06 & 16.01 & 15.52 & $\mathrm{Ar}$ \\
\hline 40 & 53632.88 & -64420.9 & 12.86 & 11.32 & 10.55 & $\mathrm{Ar}$ \\
\hline 41 & 53619.54 & -64414.9 & 13.08 & 12.53 & 12.24 & A0 \\
\hline 42 & 53632.09 & -64414.2 & 17.96 & 16.46 & 15.78 & $\mathrm{Ar}$ \\
\hline 43 & 53609.81 & -64409.3 & 17.39 & 15.86 & 15.14 & $\mathrm{Ar}$ \\
\hline 44 & 53607.19 & -64408.8 & 19.04 & 16.90 & 15.91 & $\mathrm{Ar}$ \\
\hline 45 & 53636.38 & -64408.1 & 18.99 & 17.84 & 16.95 & B \\
\hline 46 & 53613.28 & -64402.4 & 18.08 & 16.14 & 15.18 & $\mathrm{Ar}$ \\
\hline 47 & 53611.37 & -64400.1 & 16.69 & 15.23 & 14.55 & $\mathrm{Ar}$ \\
\hline 48 & 53613.45 & -64354.7 & 18.00 & 16.88 & 16.22 & B \\
\hline 49 & 53629.06 & -64351.5 & 17.83 & 15.98 & 15.11 & $\mathrm{Ar}$ \\
\hline 50 & 53626.77 & -64343.4 & 13.84 & 11.94 & 11.07 & $\mathrm{Ar}$ \\
\hline 51 & 53612.69 & -64334.0 & 15.81 & 14.35 & 13.64 & $\mathrm{Ar}$ \\
\hline
\end{tabular}


TABLE 1-Continued

\begin{tabular}{lcccccc}
\hline \hline Source & \multicolumn{2}{c}{ Position } & $\begin{array}{c}J \\
(\mathrm{mag})\end{array}$ & $\begin{array}{c}H \\
(\mathrm{mag})\end{array}$ & $\begin{array}{c}K_{s} \\
(\mathrm{mag})\end{array}$ & $\mathrm{Group}^{\mathrm{a}}$ \\
\cline { 2 - 3 } & $\alpha_{\mathrm{J} 2000.0}$ & $\delta_{\mathrm{J} 2000.0}$ & & \\
\hline 52 & 53615.27 & -64330.8 & 19.22 & 17.02 & 16.01 & $\mathrm{Ar}$ \\
53 & 53636.63 & -64323.2 & 15.72 & 14.42 & 13.88 & $\mathrm{Ar}$ \\
54 & 53634.28 & -64323.2 & 13.17 & 12.51 & 12.33 & $\mathrm{~A} 0$ \\
55 & 53627.59 & -64322.2 & 18.54 & 16.69 & 15.92 & $\mathrm{Ar}$ \\
56 & 53630.18 & -64320.4 & 17.19 & 15.77 & 15.14 & $\mathrm{Ar}$ \\
57 & 53620.52 & -64318.1 & 16.91 & 16.39 & 16.10 & $\mathrm{~A} 0$ \\
58 & 53607.34 & -64307.6 & 12.89 & 11.88 & 11.42 & $\mathrm{Ar}$ \\
59 & 53635.42 & -64307.5 & 18.69 & 17.16 & 16.50 & $\mathrm{Ar}$ \\
60 & 53625.94 & -64302.2 & 13.85 & 13.01 & 12.55 & $\mathrm{Ar}$ \\
61 & 53635.76 & -64249.9 & 13.48 & 12.38 & 11.93 & $\mathrm{Ar}$ \\
62 & 53636.87 & -64249.4 & 19.10 & 17.02 & 16.07 & $\mathrm{Ar}$ \\
63 & 53607.61 & -64246.6 & 18.14 & 16.48 & 15.73 & $\mathrm{Ar}$ \\
64 & 53630.23 & -64246.1 & 13.31 & 11.81 & 11.09 & $\mathrm{Ar}$ \\
65 & 53612.83 & -64234.6 & 19.06 & 17.26 & 16.30 & $\mathrm{Ar}$ \\
66 & 53635.14 & -64218.6 & 18.25 & 16.66 & 16.00 & $\mathrm{Ar}$ \\
67 & 53630.53 & -64203.1 & 14.99 & 14.44 & 14.16 & $\mathrm{~A} 0$ \\
68 & 53620.82 & -64156.3 & 19.52 & 17.53 & 16.71 & $\mathrm{Ar}$ \\
69 & 53632.13 & -64151.6 & 16.78 & 15.54 & 15.03 & $\mathrm{Ar}$ \\
70 & 53617.49 & -64146.1 & 18.28 & 17.08 & 16.42 & $\mathrm{Ar}$ \\
71 & 53621.96 & -64142.0 & 12.81 & 12.22 & 11.93 & $\mathrm{~A} 0$ \\
72 & 53635.81 & -64141.3 & 16.95 & 16.38 & 16.05 & $\mathrm{~A} 0$ \\
73 & 53618.80 & -64128.9 & 18.06 & 16.16 & 15.30 & $\mathrm{Ar}$ \\
74 & 53635.37 & -64129.2 & 16.08 & 14.84 & 14.33 & $\mathrm{Ar}$ \\
75 & 53619.23 & -64118.2 & 16.69 & 15.16 & 14.46 & $\mathrm{Ar}$ \\
76 & 53631.52 & -64113.6 & 12.72 & 11.98 & 11.78 & $\mathrm{~A} 0$ \\
\hline
\end{tabular}

NOTES.-Units of right ascension are hours, minutes, and seconds, and units of declination are degrees, arcminutes, and arcseconds. Positions are from the $J-H-K_{s}$ image (Fig. 1).

${ }^{a}$ Classification based on a color-color diagram (see Section 4.2). 
TABle 2

Polarimetry of Point-like Sources in the HH 1-2 Field

\begin{tabular}{|c|c|c|c|c|c|c|}
\hline Source & $\begin{array}{l}P_{J} \\
(\%)\end{array}$ & $\begin{array}{l}P_{H} \\
(\%)\end{array}$ & $\begin{array}{c}P_{K_{S}} \\
(\%)\end{array}$ & $\begin{array}{l}\theta_{J} \\
\left(^{\circ}\right)\end{array}$ & $\begin{array}{l}\theta_{H} \\
\left({ }^{\circ}\right)\end{array}$ & $\begin{array}{c}\theta_{K_{S}} \\
\left({ }^{\circ}\right)\end{array}$ \\
\hline 1 & $<12.2$ & $<6.2$ & $<20.4$ & $\cdots$ & & $\cdots$ \\
\hline 2 & $<9.6$ & $3.6 \pm 1.0$ & $<9.5$ & $\ldots$ & $113.6 \pm 8.0$ & $\ldots$ \\
\hline 3 & $40.5 \pm 8.5$ & $<10.8$ & $<32.8$ & $109.2 \pm 5.9$ & & \\
\hline 4 & $2.74 \pm 0.08$ & $2.27 \pm 0.04$ & $1.55 \pm 0.09$ & $97.7 \pm 0.8$ & $95.7 \pm 0.5$ & $83.3 \pm 1.6$ \\
\hline 5 & $<9.4$ & $<4.4$ & $<14.4$ & $\ldots$ & $\ldots$ & $\ldots$ \\
\hline 6 & $31.3 \pm 10.2$ & $5.7 \pm 1.8$ & $<12.7$ & $51.1 \pm 8.9$ & $111.8 \pm 8.5$ & \\
\hline 7 & $2.84 \pm 0.17$ & $2.23 \pm 0.09$ & $1.1 \pm 0.3$ & $121.3 \pm 1.7$ & $121.4 \pm 1.1$ & $110.8 \pm 7.0$ \\
\hline 8 & $0.30 \pm 0.04$ & $0.44 \pm 0.03$ & $0.42 \pm 0.08$ & $108.7 \pm 3.7$ & $120.8 \pm 2.0$ & $94.9 \pm 5.4$ \\
\hline 9 & $0.33 \pm 0.07$ & $0.70 \pm 0.05$ & $<0.5$ & $116.2 \pm 6.3$ & $109.7 \pm 2.1$ & $\ldots$ \\
\hline 10 & $10.97 \pm 0.06$ & $9.40 \pm 0.03$ & $7.30 \pm 0.04$ & $148.6 \pm 0.1$ & $147.7 \pm 0.1$ & $147.9 \pm 0.1$ \\
\hline 11 & $4.3 \pm 0.7$ & $3.4 \pm 0.3$ & $<4.3$ & $143.6 \pm 4.8$ & $127.9 \pm 2.9$ & $\ldots$ \\
\hline 12 & $2.4 \pm 0.6$ & $2.2 \pm 0.3$ & $<2.7$ & $119.8 \pm 7.1$ & $116.0 \pm 3.6$ & $\ldots$ \\
\hline 13 & $<13.6$ & $<5.0$ & $<13.2$ & $\ldots$ & $\ldots$ & $\ldots$ \\
\hline 14 & $6.2 \pm 0.9$ & $4.1 \pm 0.5$ & $<5.2$ & $118.6 \pm 4.0$ & $116.9 \pm 3.6$ & $\ldots$ \\
\hline 15 & $1.8 \pm 0.6$ & $2.1 \pm 0.2$ & $<2.1$ & $122.2 \pm 8.8$ & $125.3 \pm 3.2$ & $\ldots$ \\
\hline 16 & $<8.4$ & $2.9 \pm 0.8$ & $<5.9$ & $\ldots$ & $121.7 \pm 7.7$ & $\ldots$ \\
\hline 17 & $7.2 \pm 2.1$ & $3.1 \pm 0.3$ & $2.8 \pm 0.6$ & $127.8 \pm 7.9$ & $133.5 \pm 3.2$ & $119.8 \pm 5.6$ \\
\hline 18 & $4.5 \pm 0.5$ & $3.3 \pm 0.2$ & $3.2 \pm 0.7$ & $115.5 \pm 3.0$ & $118.7 \pm 2.1$ & $123.8 \pm 6.3$ \\
\hline 19 & $<30.2$ & $<7.0$ & $<19.2$ & $\ldots$ & $\ldots$ & $\ldots$ \\
\hline 20 & $<10.2$ & $7.3 \pm 1.2$ & $<8.8$ & $\ldots$ & $132.1 \pm 4.7$ & $\ldots$ \\
\hline 21 & $7.7 \pm 0.6$ & $6.07 \pm 0.15$ & $3.9 \pm 0.2$ & $20.3 \pm 2.2$ & $21.0 \pm 0.7$ & $14.0 \pm 1.7$ \\
\hline 22 & $<2.7$ & $2.8 \pm 0.5$ & $<6.4$ & $\ldots$ & $118.2 \pm 5.3$ & $\ldots$ \\
\hline 23 & $<1.2$ & $<0.9$ & $<3.4$ & $\ldots$ & & 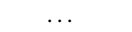 \\
\hline 24 & $<8.3$ & $4.4 \pm 0.4$ & $5.0 \pm 0.8$ & $\ldots$ & $84.0 \pm 2.6$ & $82.6 \pm 4.5$ \\
\hline 25 & $6.9 \pm 0.7$ & $5.00 \pm 0.10$ & $3.21 \pm 0.16$ & $100.3 \pm 2.9$ & $106.6 \pm 0.6$ & $108.5 \pm 1.4$ \\
\hline 26 & $0.67 \pm 0.17$ & $0.96 \pm 0.12$ & $<1.1$ & $110.0 \pm 7.1$ & $126.0 \pm 3.6$ & \\
\hline 27 & $5.89 \pm 0.18$ & $4.03 \pm 0.03$ & $2.59 \pm 0.03$ & $114.6 \pm 0.9$ & $113.5 \pm 0.2$ & $112.5 \pm 0.3$ \\
\hline 28 & $7.3 \pm 1.6$ & $4.5 \pm 0.5$ & $<3.5$ & $118.9 \pm 5.9$ & $127.4 \pm 2.9$ & \\
\hline 29 & $0.51 \pm 0.05$ & $0.74 \pm 0.04$ & $0.53 \pm 0.09$ & $118.9 \pm 3.0$ & $112.8 \pm 1.5$ & $99.6 \pm 5.0$ \\
\hline 30 & $0.62 \pm 0.20$ & $0.65 \pm 0.16$ & $2.4 \pm 0.6$ & $107.3 \pm 8.7$ & $123.3 \pm 6.7$ & $65.8 \pm 6.6$ \\
\hline 31 & $4.8 \pm 1.3$ & $<2.0$ & $7.8 \pm 2.3$ & $115.5 \pm 7.5$ & $\ldots$ & $137.9 \pm 8.1$ \\
\hline 32 & $13.6 \pm 2.5$ & $5.2 \pm 0.4$ & $3.5 \pm 0.8$ & $79.0 \pm 5.1$ & $82.6 \pm 2.2$ & $72.3 \pm 6.3$ \\
\hline 33 & $<9.6$ & $5.9 \pm 0.9$ & $<5.6$ & $\ldots$ & $114.2 \pm 4.4$ & $\ldots$ \\
\hline 34 & $6.5 \pm 0.5$ & $5.2 \pm 0.2$ & $4.1 \pm 0.5$ & $126.0 \pm 2.2$ & $115.1 \pm 1.1$ & $130.4 \pm 3.3$ \\
\hline 35 & $0.71 \pm 0.05$ & $0.83 \pm 0.03$ & $0.65 \pm 0.06$ & $71.6 \pm 2.1$ & $71.3 \pm 1.1$ & $70.9 \pm 2.8$ \\
\hline 36 & $1.49 \pm 0.10$ & $1.12 \pm 0.04$ & $0.68 \pm 0.08$ & $118.0 \pm 2.0$ & $109.5 \pm 1.1$ & $109.1 \pm 3.3$ \\
\hline 37 & $<3.5$ & $<1.0$ & $1.7 \pm 0.5$ & $\ldots$ & $\ldots$ & $118.8 \pm 8.1$ \\
\hline 38 & $2.9 \pm 0.8$ & $2.3 \pm 0.2$ & $1.8 \pm 0.4$ & $124.5 \pm 8.1$ & $111.6 \pm 2.7$ & $109.0 \pm 6.3$ \\
\hline 39 & $3.9 \pm 1.0$ & $3.2 \pm 0.5$ & $<5.7$ & $107.8 \pm 6.7$ & $113.1 \pm 4.5$ & \\
\hline 40 & $1.59 \pm 0.05$ & $1.38 \pm 0.03$ & $0.93 \pm 0.03$ & $132.8 \pm 0.9$ & $128.0 \pm 0.5$ & $126.7 \pm 0.9$ \\
\hline 41 & $0.57 \pm 0.05$ & $0.65 \pm 0.04$ & $0.48 \pm 0.09$ & $115.9 \pm 2.7$ & $114.8 \pm 1.6$ & $112.8 \pm 5.4$ \\
\hline 42 & $<8.2$ & $5.0 \pm 1.2$ & $<7.5$ & $\ldots$ & $114.7 \pm 6.4$ & $\ldots$ \\
\hline 43 & $<4.3$ & $2.4 \pm 0.5$ & $<3.8$ & $\ldots$ & $107.8 \pm 5.3$ & $\ldots$ \\
\hline 44 & $<26.1$ & $5.6 \pm 1.7$ & $<9.5$ & $\ldots$ & $126.2 \pm 8.2$ & $\ldots$ \\
\hline 45 & $<15.0$ & $<10.0$ & $<20.7$ & $\ldots$ & $\ldots$ & $\ldots$ \\
\hline 46 & $10.9 \pm 2.5$ & $5.4 \pm 0.7$ & $<4.1$ & $82.9 \pm 6.3$ & $107.1 \pm 3.9$ & $\ldots$ \\
\hline 47 & $3.5 \pm 0.7$ & $2.0 \pm 0.3$ & $<2.3$ & $84.2 \pm 5.9$ & $113.6 \pm 3.7$ & $\ldots$ \\
\hline 48 & $17.8 \pm 2.5$ & $8.8 \pm 1.3$ & $<10.1$ & $149.5 \pm 4.0$ & $133.9 \pm 4.1$ & $\ldots$ \\
\hline 49 & $<7.5$ & $5.2 \pm 0.6$ & $<3.8$ & & $104.6 \pm 3.2$ & $\ldots$ \\
\hline 50 & $1.64 \pm 0.11$ & $1.09 \pm 0.04$ & $0.56 \pm 0.04$ & $90.3 \pm 1.8$ & $91.8 \pm 1.0$ & $109.0 \pm 2.0$ \\
\hline 51 & $4.7 \pm 0.4$ & $3.03 \pm 0.14$ & $1.8 \pm 0.3$ & $86.9 \pm 2.1$ & $95.3 \pm 1.3$ & $99.2 \pm 4.9$ \\
\hline 52 & $<21.9$ & $<4.2$ & $<8.7$ & $\ldots$ & $\ldots$ & $\ldots$ \\
\hline 53 & $3.6 \pm 0.3$ & $2.56 \pm 0.16$ & $1.7 \pm 0.4$ & $131.5 \pm 2.7$ & $122.8 \pm 1.8$ & $137.9 \pm 6.5$ \\
\hline 54 & $0.66 \pm 0.05$ & $0.70 \pm 0.04$ & $0.63 \pm 0.10$ & $125.6 \pm 2.4$ & $120.7 \pm 1.4$ & $110.7 \pm 4.6$ \\
\hline 55 & $<17.8$ & $8.3 \pm 1.4$ & $10.3 \pm 2.8$ & $\ldots$ & $106.4 \pm 4.8$ & $128.4 \pm 7.6$ \\
\hline 56 & $<3.7$ & $3.4 \pm 0.5$ & $<3.7$ & $\ldots$ & $110.1 \pm 4.1$ & $\ldots$ \\
\hline 57 & $<3.1$ & $2.6 \pm 0.8$ & $<9.4$ & $\ldots$ & $104.5 \pm 8.1$ & $\ldots$ \\
\hline 58 & $4.14 \pm 0.05$ & $2.54 \pm 0.03$ & $1.39 \pm 0.06$ & $92.9 \pm 0.3$ & $95.2 \pm 0.3$ & $103.5 \pm 1.2$ \\
\hline 59 & $<13.7$ & $<4.9$ & $<13.5$ & $\ldots$ & $\ldots$ & $\ldots$ \\
\hline 60 & $<3.6$ & $<2.8$ & $<2.5$ & $\ldots$ & $\ldots$ & $\ldots$ \\
\hline 61 & $2.31 \pm 0.07$ & $1.70 \pm 0.04$ & $1.08 \pm 0.07$ & $130.5 \pm 0.8$ & $125.4 \pm 0.6$ & $114.5 \pm 1.9$ \\
\hline 62 & $<17.7$ & $7.0 \pm 1.5$ & $<9.8$ & $\ldots$ & $135.6 \pm 5.9$ & $\ldots$ \\
\hline 63 & $<10.6$ & $<2.8$ & $<6.6$ & $\ldots$ & $\ldots$ & $\ldots$ \\
\hline 64 & $1.47 \pm 0.06$ & $1.21 \pm 0.03$ & $0.81 \pm 0.04$ & $126.7 \pm 1.1$ & $129.3 \pm 0.6$ & $138.5 \pm 1.3$ \\
\hline 65 & $<17.3$ & $<4.7$ & $<11.3$ & & $\ldots$ & $\ldots$ \\
\hline 66 & $13.7 \pm 3.1$ & $<3.8$ & $<8.8$ & $120.3 \pm 6.3$ & $\ldots$ & $\ldots$ \\
\hline
\end{tabular}


TABLE 2-Continued

\begin{tabular}{lcccccc}
\hline \hline Source & $\begin{array}{c}P_{J} \\
(\%)\end{array}$ & $\begin{array}{c}P_{H} \\
(\%)\end{array}$ & $\begin{array}{c}P_{K_{S}} \\
(\%)\end{array}$ & $\begin{array}{c}\theta_{J} \\
\left({ }^{\circ}\right)\end{array}$ & $\begin{array}{c}\theta_{H} \\
\left({ }^{\circ}\right)\end{array}$ & $\begin{array}{c}\theta_{K_{S}} \\
\left({ }^{\circ}\right)\end{array}$ \\
\hline 67 & $<0.5$ & $0.52 \pm 0.15$ & $2.0 \pm 0.5$ & $\ldots$ & $98.6 \pm 8.1$ & $124.5 \pm 7.3$ \\
68 & $<33.9$ & $<7.5$ & $<16.2$ & $\ldots$ & $\ldots$ & $\ldots$ \\
69 & $7.2 \pm 0.8$ & $4.3 \pm 0.4$ & $<3.4$ & $115.7 \pm 3.2$ & $118.6 \pm 2.6$ & $\ldots$ \\
70 & $9.9 \pm 3.1$ & $<4.6$ & $13.1 \pm 4.3$ & $35.6 \pm 8.6$ & $\ldots$ & $100.9 \pm 8.9$ \\
71 & $0.43 \pm 0.05$ & $0.62 \pm 0.03$ & $0.45 \pm 0.07$ & $135.8 \pm 3.0$ & $134.6 \pm 1.4$ & $134.3 \pm 4.6$ \\
72 & $<4.0$ & $<2.5$ & $<9.2$ & $\ldots$ & $\ldots$ & $\ldots$ \\
73 & $<10.0$ & $5.1 \pm 0.8$ & $<5.2$ & $\ldots$ & $101.3 \pm 4.2$ & $\ldots$ \\
74 & $2.7 \pm 0.7$ & $3.5 \pm 0.2$ & $<2.2$ & $120.4 \pm 7.5$ & $120.9 \pm 2.0$ & $\ldots$ \\
75 & $8.8 \pm 1.4$ & $4.6 \pm 0.4$ & $4.6 \pm 1.0$ & $88.1 \pm 4.3$ & $101.0 \pm 2.6$ & $123.3 \pm 6.3$ \\
76 & $0.58 \pm 0.06$ & $0.53 \pm 0.03$ & $<0.34$ & $110.0 \pm 2.8$ & $105.0 \pm 1.8$ & $\ldots$ \\
\hline
\end{tabular}

Notes.-For sources with $P / \delta P<3$, the $3 \delta P$ upper limits are listed.

TABLE 3

Optical Jets IN THE HH 1-2 FiELD

\begin{tabular}{lrcc}
\hline \hline \multicolumn{1}{c}{ Object } & P.A. $^{\text {a }}$ & Driving Source & References \\
\hline HH 1-2 & $148^{\circ}$ & VLA 1 & Pravdo et al. 1985 \\
HH 35 & $149^{\circ}$ & V380 Ori & Strom et al. 1986 \\
HH 144-145 & $82^{\circ}$ & VLA 2 & Reipurth et al. 1993 \\
HH 146 & $6^{\circ}$ & VLA 4 & Reipurth et al. 1993 \\
HH 147 & $50^{\circ}$ & N $^{3}$ SK 50 & Eislöffel et al. 1994 \\
HH 148 & $56^{\circ}$ & V380 Ori & Strom et al. 1986 \\
SMZ 61 & $171^{\circ}$ & VLA 3 & Stanke et al. 2002
\end{tabular}

${ }^{a}$ Position angle of the outflow axis. Typical uncertainty is $10^{\circ}$. 